\title{
Collision Resolution for Random Access
}

\author{
Mohammad Kazemi, Member, IEEE, Tolga M. Duman, Fellow, IEEE, and Muriel Médard, Fellow, IEEE
}

\begin{abstract}
As a building block toward a simple and scalable solution for massive random access, we introduce collisionresolution algorithms using successive interference cancellation (SIC) based on the received signals, with no need for any coordination or codebook differentiation. We first consider twouser multiple access with the ZigZag algorithm. We prove that the original ZigZag and a modified version of it, called double-zipper ZigZag, attain the same performance as the optimal coordinated time-sharing in the high signal to noise ratio (SNR) regime, even in the presence of channel state information (CSI) errors. We then extend the results to the case of arbitrary number of users employing delay-domain processing. Specifically, we introduce delay-domain zero forcing and its regularized version, which are able to cancel and suppress the interference among users, respectively. By obtaining a post-processing system model and characterizing the accumulated noise during the decoupling process, we also derive bounds on the achievable sum-rates of the proposed algorithm for both cases of perfect and imperfect CSI. Simulation results show that the newly proposed approach have comparable performance with coordinated time-sharing at high SNRs.
\end{abstract}

Index Terms-ZigZag algorithm, collision resolution, interference cancellation, delay domain processing, massive random access.

\section{INTRODUCTION}

With the exponentially increasing number of internet-ofthings (IoT) devices and the rise of machine-to-machine (M2M) communications, simple and scalable solutions for the problem of massive random access are of paramount importance. In this setting, a very large number of users, of which only a small subset are active at any given time, wish to communicate their messages to a common receiver in an uncoordinated fashion. Two parallel approaches have been developed in the previous literature to address the problem of multiple access communications. The first approach follows the footsteps of Ahlswede [2] and Liao [3], and considers multiple access channels (MACs) for which codebooks of individual users are distinct and the channels are perfectly synchronized. The receiver, having access to the users' codebooks, can decode the individual messages using different techniques. The rate region achievable via this approach is named as the Cover-Wyner rate region [4]. Considering Gaussian noise channels, at high signal to noise ratios (SNRs), the rate region becomes nearly triangular and approaches the time-sharing region; while at low SNRs, it becomes a rectangular one

This work was supported by the Scientific and Technological Research Council of Turkey (TÜBITTAK) under the Grant 119E589. This paper was presented in part at the 2020 IEEE International Conference on Communications (ICC). [1].

M. Kazemi and T. M. Duman are with the Department of Electrical and Electronics Engineering, Bilkent University, Bilkent, Ankara, 06800, Turkey (email: kazemi@ee.bilkent.edu.tr, duman@ee.bilkent.edu.tr).

M. Médard is with the Department of EECS, MIT, Cambridge, MA (email: medard@mit.edu).
[5], [6]. While it is possible to achieve the MAC capacity via this approach, it does not scale easily beyond a few users. Recently, compressed sensing techniques have been employed to alleviate this problem. The readers are referred to [7] and the references therein for related algorithms to estimate the number of users, channel coefficients, and users' packets in a random access setup. Even though it is not impossible to assign distinct codebooks to a massive number of users and keep track of the transmitting ones at the receiver, synchronization issues makes it very hard to implement this approach in practice. Some works also employ the spatial dimension alongside the compressed sensing techniques in order to increase the detection accuracy [8].

The second approach which has originated from the University of Hawaii in early 1970s is the simple ALOHA protocol [9] and its slotted variant [10], which are based on collision avoidance ignoring the channel coding problem. As the approach is based on collision avoidance, the colliding packets are discarded at the receiver. While this approach is scalable in terms of the number of users, its overall throughput performance is highly inferior. More recent literature considers some extensions of ALOHA along with successive interference cancellation (SIC) to improve its throughput. For instance, in [11], a combination of packet erasure correcting codes and SIC, called coded slotted ALOHA, is developed. The scheme exploits a bipartite graph representation of the SIC process, resembling iterative decoding of generalized lowdensity parity-check (LDPC) codes over the erasure channel to optimize the selection probabilities of the component erasure correcting codes through a density evolution analysis. In diversity ALOHA [12], the authors utilize time and frequency diversities to transmit multiple copies of the users' packets, which results in better delay performance compared to the simple slotted ALOHA under light traffic. Employing this repetition strategy, contention resolution diversity slotted ALOHA (CRDSA) [13] considers grouping a number of slots as frames in which each user sends two copies of its packet at the physical layer. On the collision channel, SIC among slots is then adopted to resolve the collisions given the received signals in the entire frame. Liva in [14] proposes to vary the number of copies being transmitted according to a probability distribution resulting in irregular repetition slotted ALOHA (IRSA). He introduces an iterative process to analyze the asymptotic performance of the system for a fixed repetition distribution similar to the density evolution based analysis of LDPC codes. In [15], the authors design aggregate preamble Zadoff-Chu sequences in order to reduce the probability of preamble collision.

A recent information theoretic formulation for the massive random access problem is developed by Polyanskiy [16]. Assuming that the users utilize the same codebook, he defines 
a $K_{a}$-user MAC code as a collection of norm-constrained vectors, where the noisy sum of any $K_{a}$ of them can be decoded with a certain probability of error. Ref. [16] also presents some bounds on the capacity of the massive random access systems. However, in this formulation too, the users' transmissions are assumed to be fully synchronized, and it appears difficult to achieve these information theoretic limits in practice [17]-[19]. In [17], a low complexity coding scheme is proposed for massive random access setup, which is based on a combination of compute-and-forward scheme and coding for a binary-adder channel. The code is a concatenation of two codes: an inner binary linear code, used to decode the modulo2 sum of all the codewords transmitted, and an outer code whose role is to identify the individual codewords that make up the sum. In [18], the message is split into two parts where one part is encoded using spreading sequences that are designed to be decodable using a compressed sensing type decoder, and the other one is encoded using LDPC codes. Also the users repeat their messages among different slots via a deterministic function of the message content (independent of the user), which is then exploited via successive interference cancellation to improve the system performance. The results are found to be significantly improved compared to the approach of [17], at the cost of high computational complexity. Binary chirps (codewords of the second order Reed Muller code) are utilized as compressed sensing codes in [19], with the main advantage of reducing complexity.

There are several results which build upon asynchronism of data transmission in MAC scenarios and introduce decoding algorithms based on the delayed received signals. In [20], the artificial packet delays are assigned to each user by the receiver (base station) in a coordinated manner. Then, exploiting the introduced delays, the receiver decouples the packets using SIC. In [21], each user packet is repeated a certain number of times, interleaved and multiplied with a signature sequence known to the receiver. The receiver decodes the sum of delayed packets using a window-based iterative SIC. These works need some level of coordination either in the form of delay assignment or the knowledge of signature sequences at the receiver.

With the aim of facilitating massive random access in a practical manner, we consider the use of interference cancellation techniques based on the received signal itself in a similar manner as in the ZigZag algorithm [22]. ZigZag is an interference cancellation algorithm proposed by Katabi in [22], which has already been implemented in practice. The basic idea is to transmit the packets multiple times in such a way that different replicas experience different delays at the receiver. This could be due to the differences in channel delays or may be introduced artificially by proper randomization at the transmitters. The receiver performs interference cancellation based on the received signals only, i.e., without the need for decoding the messages. Once the interference is canceled, each user's message is decoded separately. With this methodology, the users can adopt the same codebooks (designed for single user communications), i.e., this scheme can be used as a building block for massive random access.

Some aspects of the ZigZag algorithm have been stud- ied in the previous literature. In [23], the authors compare the throughput performances of ALOHA and carrier-sense multiple access (CSMA) schemes with ZigZag. An algebraic representation of collisions which views each collision as a linear combination of the original packets is presented in [24]. This scheme outperforms not only the ALOHA-type schemes from a delay perspective, but also the centralized scheduling solutions. In [25], two ACK policies are proposed that stabilize the random access system with ZigZag. In [26], the authors show that ZigZag can be seen as an instance of belief propagation in the high SNR regime. Building on this observation, they present a simple soft-decoding version, called SigSag. In [27], ZigZag decodable (ZD) codes are proposed for distributed storage systems. A fountain coding system based on ZD codes is proposed in [28]. In [29], unlike the original ZigZag, the received packets are decoupled from both sides, resulting in fewer decoupling steps. We refer to this scheme as double-zipper ZigZag in this paper. To deal with the error floor problem in a frameless structure, the authors also devise a scheme with two-bit feedback called enhanced ZigZag decodable frameless ALOHA (E-ZDFA). The asymptotic throughput and packet loss rate of the EZDFA scheme are derived in [30] in a noise-free system model with unit channel gains. Unlike [29] and [30], we consider the effects of both noise and non-unit channel gains in this paper and determine information theoretic bounds on the achievable sum-rates, depicting that our proposed algorithms are asymptotically optimal. In addition, while [29] and [30] are able to resolve the collision of two users, we also propose a scheme to resolve the collision of more than two users.

In this paper, we present simple and scalable collision resolution algorithms based on interference cancellation with the received signals that can be utilized as building blocks toward simple and effective solutions to the problem of massive random access. The contributions of the paper are fourfold:

- For a two-user MAC, we characterize noise coefficient matrices of both original and double-zipper ZigZag algorithms, which are then used to obtain bounds on their performance.

- For arbitrary number of users, we propose delay-domain processing algorithms, namely, delay-domain zero forcing (DD-ZF) and regularized DD-ZF, which perform interference cancellation and interference suppression, respectively, based on the received (noisy) signals.

- We derive upper and lower bounds on the performance of the proposed algorithms in the presence of imperfect channel state information (CSI).

- We show that the gap between the lower bound on the achievable sum-rate of the proposed scheme and the upper bound on the time-sharing sum-rate becomes a constant at high SNRs. In other words, the performance of the proposed scheme asymptotically approaches that of optimal coordinated time-sharing based solutions for arbitrary user delays. We also show that a similar result holds for the ZigZag algorithms.

The rest of the paper is organized as follows. The system model is introduced in Section II. In Section III, we present 


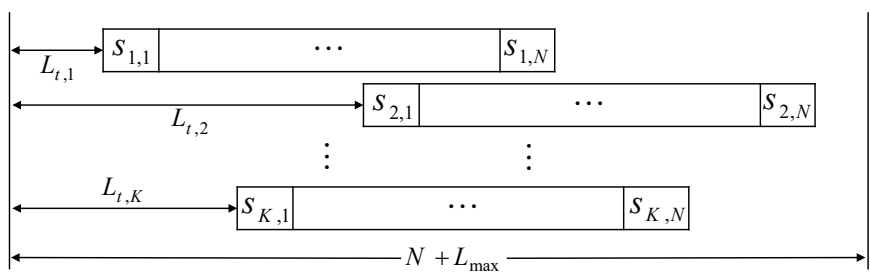

Fig. 1: Received delayed packets in the $t$-th time-interval.

several capacity expressions for the benchmark schemes. In Section IV, a short explanation of ZigZag decoupling process is given along with a characterization of the resulting noise. By providing bounds on the achievable sum-rate of the ZigZag algorithms, we prove their asymptotic optimality even with imperfect CSI. Delay-domain processing algorithms, DD-ZF and regularized DD-ZF, are introduced in Section V. We also provide bounds on the performance of these algorithms in the presence of imperfect CSI. Numerical examples are provided in Section VI, and finally, the paper is concluded in Section VII.

\section{System Model}

We have $K$ users, each interested in transmitting a packet of length $N$ to a common receiver. Since there is no coordination among the users or the receiver, collisions occur. To resolve the collisions, we rely on delay differences in the users' signals. To be more precise, there are $T$ time-intervals. In each time-interval, the users simultaneously send their packets without synchronization, hence the packets experience different delays. Note that while the delays may be naturally occurring, they may also be introduced by randomizing the packet transmission times. The receiver employs interference cancellation techniques to decouple the users' packets. To simplify the exposition, we also assume that the received packets are synchronous at the symbol level; i.e., although time of arrivals (delays) of the packets are different, they are integer multiples of the symbol period. We assume that the receiver has a perfect knowledge of the number of users and their packet delays in each time-interval and an imperfect knowledge of their channel gains, which are constant over the $T$ time-intervals. To motivate this channel model, consider, for example, a mobile user moving at the speed of $100 \mathrm{~km} / \mathrm{h}$ and transmitting at $5 \mathrm{GHz}$ with a data rate of $50 \mathrm{Mbps}$ and packet length of 5000 bits; the coherence time is about $1 \mathrm{~ms}$. Hence, the channel is constant for a length of 10 packets (10 timeintervals). In other words, the assumption of channel gains being constant for a few packet lengths is practical.

The number of active users and the channel gains can be jointly estimated, employing techniques such as the minimum description length (MDL) criterion [31] and the compressed sensing [7] based algorithms. The receiver can estimate the user delays by first employing energy detection techniques to estimate the delays and then trying the possible combinations to associate the delays to the users. A more scalable approach would be for the users to utilize random sequences in their preambles (and/or postambles) in an uncoordinated fashion. They can employ different sequences in different transmissions; they just need to use the same sequence for the $T$

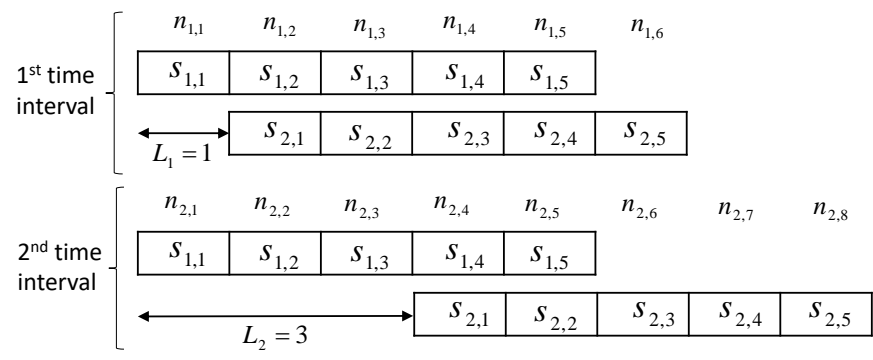

Fig. 2: Received delayed packets in a two-user system with $N=5$ and $L=\left[\begin{array}{ll}0 & 1 \\ 0 & 3\end{array}\right]$.

time-intervals. There are other approaches in the literature for multiple delay estimation as well. For instance, [31] presents a maximum likelihood based algorithm as well as a low-complexity variational inference based algorithm for estimation of the delays in a random access setup.

Let $\mathbf{L}$ be the $T \times K$ delay matrix with the $(t, k)$-th entry, $L_{t, k}$, being the time delay of the $t$-th packet of the $k$-th user from the start of $t$-th time-interval. The time delays are with respect to data symbols, i.e., they are non-negative integers between 0 and $L_{\max }$ with $L_{\max }$ being the maximum possible delay. An illustration of the received nonaligned packets in the $t$-th time-interval is given in Fig. 1. where $s_{i, j}$ is the $j$-th symbol of the $i$-th user. Assuming that the channel gain of each user is constant over the $T$ time-intervals, the received signals in the delay domain can be expressed as

$$
\mathbf{y}(D)=\mathbf{L}(D) \mathbf{H s}(D)+\mathbf{n}(D)
$$

where $D$ is the delay operator, $\mathbf{L}(D)$ is the $T \times K$ delay matrix with the $(t, k)$-th entry being $\mathbf{L}_{t, k}(D)=D^{L_{t, k}}, \mathbf{H}$ is the $K \times K$ diagonal channel matrix with the $k$-th diagonal entry being the $k$-th user's channel coefficient $h_{k}, \mathbf{y}(D)=$ $\left[y_{1}(D), \ldots, y_{T}(D)\right]^{\mathrm{T}}$ with $y_{t}(D)=\sum_{j=1}^{N+L_{\max }} y_{t, j} D^{j-1}$, where $y_{t, j}$ is the $j$-th received signal in the $t$-th time-interval, $\mathbf{s}(D)=$ $\left[s_{1}(D), \ldots, s_{K}(D)\right]^{\mathrm{T}}$ with $s_{k}(D)=\sum_{j=1}^{N} s_{k, j} D^{j-1}$, where $s_{k, j}$ is the $j$-th symbol of the $k$-th user. Since we consider bandpass signals, the transmitted symbols as well as the channel gains are complex. In $\left[1, \mathbf{n}(D)=\left[n_{1}(D), \ldots, n_{T}(D)\right]^{\mathrm{T}}\right.$ with $n_{t}(D)=\sum_{j=1}^{N+L_{\max }} n_{t, j} D^{j-1}$, where $n_{t, j}$ is the $j$-th additive white Gaussian noise (AWGN) term in the $t$-th time-interval at the receiver, independent of the other noise terms and the transmitted symbols, with zero mean and variance $\sigma_{n}^{2}$.

As an example, consider a two-user system with $N=5$ and $L=\left[\begin{array}{ll}0 & 1 \\ 0 & 3\end{array}\right]$, depicted in Fig. 2. In this case, the matrices in 11 can be written as:

$$
\begin{aligned}
& \mathbf{s}(D)=\left[\begin{array}{l}
s_{1,1}+s_{1,2} D+s_{1,3} D^{2}+s_{1,4} D^{3}+s_{1,5} D^{4} \\
s_{2,1}+s_{2,2} D+s_{2,3} D^{2}+s_{2,4} D^{3}+s_{2,5} D^{4}
\end{array}\right], \\
& \mathbf{n}(D)=\left[\begin{array}{l}
n_{1,1}+n_{1,2} D+n_{1,3} D^{2}+s_{1,4} D^{3}+n_{1,5} D^{4}+n_{1,6} D^{5} \\
n_{2,1}+n_{2,2} D+n_{2,3} D^{2}+s_{2,4} D^{3}+n_{2,5} D^{4}+n_{2,6} D^{5}+ \\
n_{2,7} D^{6}+n_{2,8} D^{7}
\end{array}\right], \\
& \mathbf{L}(D)=\left[\begin{array}{ll}
1 & D \\
1 & D^{3}
\end{array}\right], \mathbf{H}=\left[\begin{array}{ll}
h_{1} & 0 \\
0 & h_{2}
\end{array}\right] .
\end{aligned}
$$


The received signal after post-processing at the receiver (such as ZigZag) and conversion from the delay domain, $\hat{\mathbf{y}}$, can be written as:

$$
\hat{\mathbf{y}}=\mathbf{H}_{e q} \mathbf{s}+\mathbf{A n},
$$

where $\mathbf{H}_{e q}$ is a $K N \times K N$ matrix whose entries are functions of user channel coefficients and $\mathbf{A}$ is the $K N \times T\left(N+L_{\max }\right)$ noise coefficient matrix denoting the accumulated noise in the decoupling process. Note that both $\mathbf{H}_{e q}$ and $\mathbf{A}$ are determined by the employed post-processing (decoupling) scheme at the receiver Also, $\mathbf{s}=\left[\mathbf{s}_{1}^{\mathrm{T}}, \ldots, \mathbf{s}_{K}^{\mathrm{T}}\right]^{\mathrm{T}}$ with $\mathbf{s}_{k}=\left[s_{k, 1}, \ldots, s_{k, N}\right]^{\mathrm{T}}$, and $\mathbf{n}=\left[\mathbf{n}_{1}^{\mathrm{T}}, \ldots, \mathbf{n}_{T}^{\mathrm{T}}\right]^{\mathrm{T}}$ with $\mathbf{n}_{t}=\left[n_{t, 1}, \ldots, n_{t, N+L_{\max }}\right]^{\mathrm{T}}$, i.e., $\mathbf{s}$ and $\mathbf{n}$ contain all the coefficients of polynomial matrices $\mathbf{s}(D)$ and $\mathbf{n}(D)$, respectively. We will explain the matrices in (2) through an example when we discuss some decoupling strategies in Section IV. Note that (2) serves as the postprocessing system model.

\section{Benchmark Schemes}

We now present several capacity expressions, which are used for comparisons throughout the paper, assuming that the users utilize independent and identically distributed (i.i.d.) Gaussian codebooks with elements of equal power $P$.

\section{A. Perfect CSI Case}

For single-user decoding, (2) can be divided into sub-models for each user as follows

$$
\hat{\mathbf{y}}_{k}=\mathbf{H}_{e q, k} \mathbf{s}_{k}+\mathbf{H}_{e q, k}^{c} \mathbf{s}_{k}^{c}+\mathbf{A}_{k} \mathbf{n},
$$

where $\mathbf{s}_{k}^{c}=\left[\mathbf{s}_{1}^{\mathrm{T}}, \ldots, \mathbf{s}_{k-1}^{\mathrm{T}}, \mathbf{s}_{k+1}^{\mathrm{T}}, \ldots, \mathbf{s}_{K}^{\mathrm{T}}\right]^{\mathrm{T}}, \hat{\mathbf{y}}_{k}$ and $\mathbf{A}_{k}$ are obtained from the rows $(k-1) N+1$ to $k N$ of $\hat{\mathbf{y}}$ and $\mathbf{A}$, respectively, $\mathbf{H}_{e q, k}$ contains entries of $\mathbf{H}_{e q}$ between rows $(k-1) N+1$ to $k N$ and columns $(k-1) N+1$ to $k N, \mathbf{H}_{e q, k}^{c}$ is obtained from the rows $(k-1) N+1$ to $k N$ of $\mathbf{H}_{e q}$ with $\mathbf{H}_{e q, k}$ removed. As an example, consider a two-user system with $\mathbf{H}_{e q}=\left[\begin{array}{llll}4 & 2 & 5 & 4 \\ 9 & 2 & 2 & 5 \\ 2 & 8 & 8 & 4 \\ 3 & 6 & 6 & 1\end{array}\right]$ (hence $N=2$ ). Then, we have $\mathbf{H}_{e q, 1}=\left[\begin{array}{ll}4 & 2 \\ 9 & 2\end{array}\right]$, $\mathbf{H}_{e q, 2}=\left[\begin{array}{lll}3 & 6 & 6 \\ 6 & 4\end{array}\right], \mathbf{H}_{e q, 1}^{c}=\left[\begin{array}{ll}5 & 4 \\ 2 & 5\end{array}\right]$ and $\mathbf{H}_{e q, 2}^{c}=\left[\begin{array}{ll}2 & 8 \\ 3 & 6\end{array}\right]$.

Therefore, treating interference as noise, the achievable sum-rate (per channel use) of single-user decoding becomes

$$
\begin{aligned}
R_{S U} & =\frac{1}{T N} \sum_{k=1}^{K} \log \left(\frac{\left|\mathbf{R}_{n, k}+\mathbf{R}_{s, k}^{c}+\mathbf{R}_{s, k}\right|}{\left|\mathbf{R}_{n, k}+\mathbf{R}_{s, k}^{c}\right|}\right) \\
& =\frac{1}{T N} \sum_{k=1}^{K} \log \left(\frac{\left|\mathbf{A}_{k} \mathbf{A}_{k}^{\mathrm{T}}+\rho\left(\mathbf{H}_{e q, k}^{c} \mathbf{H}_{e q, k}^{c \mathrm{H}}+\mathbf{H}_{e q, k} \mathbf{H}_{e q, k}^{\mathrm{H}}\right)\right|}{\left|\mathbf{A}_{k} \mathbf{A}_{k}^{\mathrm{T}}+\rho \mathbf{H}_{e q, k}^{c} \mathbf{H}_{e q, k}^{c \mathrm{H}}\right|}\right),
\end{aligned}
$$

where $\mathbf{R}_{s, k}, \mathbf{R}_{s, k}^{c}$ and $\mathbf{R}_{n, k}$ are the covariance matrices of the signal, the interference and the noise terms of $k$-th user in (3), respectively, $\rho:=\frac{P}{\sigma_{n}^{2}}$ is the transmit SNR, and $|\mathbf{X}|$ denotes the determinant of the matrix $\mathbf{X}$.

The sum-rate capacity (per channel use) is given by

$$
C_{S C}=\log \left(1+\rho \sum_{k=1}^{K}\left|h_{k}\right|^{2}\right),
$$

while the time-sharing sum-rate capacity (per channel use) is

$$
C_{T S}=\frac{1}{K} \sum_{k=1}^{K} \log \left(1+K \rho\left|h_{k}\right|^{2}\right) .
$$

For the 2-user case, we will also use as reference the Avestimehr-Diggavi-Tse expression derived in [32] using a deterministic approach, which is within 0.5 bits per user of the capacity. It is given by

$$
C_{A D T}=\left\lceil\log \left(\rho \max \left\{\left|h_{1}\right|^{2},\left|h_{2}\right|^{2}\right\}\right)\right]^{+},
$$

where $\lceil a\rceil^{+}$denotes the smallest non-negative integer greater than or equal to $a$.

\section{B. Imperfect CSI Case}

We now assume that only the estimates of channel gains are available to the receiver, $h_{k}=\bar{h}_{k}+\tilde{h}_{k}$, where $\bar{h}_{k}$ and $\tilde{h}_{k}$ are the estimated channel gain and the channel estimation error of the $k$-th user, respectively. The channel estimation errors are modeled as independent zero-mean random variables with a known variance $\sigma_{h}^{2}$, which is inversely proportional to the SNR, i.e., $\sigma_{h}^{2}=\frac{c}{\rho}$ for some constant $c \geq 0$ [33], [34].

For imperfect CSI, 2) can be written as

$$
\hat{\mathbf{y}}=\overline{\mathbf{H}}_{e q} \mathbf{s}+\tilde{\mathbf{H}}_{e q} \mathbf{s}+\mathbf{A n},
$$

where the equivalent channel matrix is divided into known and unknown parts, i.e., $\mathbf{H}_{e q}=\overline{\mathbf{H}}_{e q}+\tilde{\mathbf{H}}_{e q}$. Note that in general, $\overline{\mathbf{H}}_{e q}$ is a function of $\bar{h}_{k}$ 's, while $\tilde{\mathbf{H}}_{e q}$ is a function of both $\bar{h}_{k}$ 's and $\tilde{h}_{k}$ 's.

For single-user decoding, 8 is divided into sub-models for each user as follows

$$
\hat{\mathbf{y}}_{k}=\overline{\mathbf{H}}_{e q, k} \mathbf{s}_{k}+\tilde{\mathbf{H}}_{e q, k} \mathbf{s}_{k}+\overline{\mathbf{H}}_{e q, k}^{c} \mathbf{s}_{k}^{c}+\tilde{\mathbf{H}}_{e q, k}^{c} \mathbf{s}_{k}^{c}+\mathbf{A}_{k} \mathbf{n},
$$

where $\overline{\mathbf{H}}_{e q, k}$ and $\tilde{\mathbf{H}}_{e q, k}$ contain entries of $\overline{\mathbf{H}}_{e q}$ and $\tilde{\mathbf{H}}_{e q}$ between rows $(k-1) N+1$ to $k N$ and columns $(k-1) N+1$ to $k N$, respectively. Also, $\overline{\mathbf{H}}_{e q, k}^{c}$ and $\tilde{\mathbf{H}}_{e q, k}^{c}$ are obtained from the rows $(k-1) N+1$ to $k N$ of $\overline{\mathbf{H}}_{e q}$ and $\tilde{\mathbf{H}}_{e q}$ with $\overline{\mathbf{H}}_{e q, k}$ and $\tilde{\mathbf{H}}_{e q, k}$ removed, respectively.

Treating uncorrelated interference as noise and using the results in [35], lower and upper bounds on the achievable sum-rate (per channel use) with single-user decoding in the presence of imperfect CSI are obtained as

$$
\begin{gathered}
R_{S U} \geq \frac{1}{T N} \sum_{k=1}^{K} \log \left(\mid\left(\tilde{\mathbf{R}}_{k}+\tilde{\mathbf{R}}_{k}^{c}+\overline{\mathbf{H}}_{e q, k}^{c} \overline{\mathbf{H}}_{e q, k}^{c \mathrm{H}}+\frac{1}{\rho} \mathbf{A}_{k} \mathbf{A}_{k}^{\mathrm{T}}\right)^{-1}\right. \\
\left.\overline{\mathbf{H}}_{e q, k} \overline{\mathbf{H}}_{e q, k}^{\mathrm{H}}+\mathbf{I}_{N} \mid\right)
\end{gathered}
$$

and

$$
\begin{gathered}
R_{S U} \leq \frac{1}{T N} \sum_{k=1}^{K} \log \left(\mid\left(\tilde{\mathbf{R}}_{k}^{c}+\overline{\mathbf{H}}_{e q, k}^{c} \overline{\mathbf{H}}_{e q, k}^{c \mathrm{H}}+\frac{1}{\rho} \mathbf{A}_{k} \mathbf{A}_{k}^{\mathrm{T}}\right)^{-1}\right. \\
\left.\left(\overline{\mathbf{H}}_{e q, k} \overline{\mathbf{H}}_{e q, k}^{\mathrm{H}}+\tilde{\mathbf{R}}_{k}\right)+\mathbf{I}_{N} \mid\right),
\end{gathered}
$$

respectively, where $\tilde{\mathbf{R}}_{k}:=\mathbb{E}\left[\tilde{\mathbf{H}}_{e q, k} \tilde{\mathbf{H}}_{e q, k}^{\mathrm{H}}\right]$ and $\tilde{\mathbf{R}}_{k}^{c}:=$ $\mathbb{E}\left[\tilde{\mathbf{H}}_{e q, k}^{c} \tilde{\mathbf{H}}_{e q, k}^{c \mathrm{H}}\right]$, where $\mathbb{E}$ denotes statistical expectation. Note 
that the case of perfect CSI is recovered when $\tilde{\mathbf{R}}_{k}=\tilde{\mathbf{R}}_{k}^{c}=\mathbf{0}$ (i.e., selecting $c=0$ ). Also, using the results in [35], lower and upper bounds on the time-sharing sum-rate capacity (per channel use) with imperfect CSI can be obtained as

$$
C_{T S} \geq \frac{1}{K} \sum_{k=1}^{K} \log \left(1+\frac{K \rho\left|\bar{h}_{k}\right|^{2}}{K c+1}\right),
$$

and

$$
C_{T S} \leq \frac{1}{K} \sum_{k=1}^{K} \log \left(1+K c+K \rho\left|\bar{h}_{k}\right|^{2}\right),
$$

respectively. Again, the case of perfect CSI is recovered with $c=0$.

\section{Two User Case: Characterization of ZigZaG}

In this section, we focus on $K=2, T=2$ case as an important special case of our system model, and consider the use of the ZigZag algorithm. The basic idea of ZigZag is to perform interference cancellation based on only the received signals at the receiver, i.e., without the need for decoding of users' messages. Once the interference is canceled out, each user's message can be decoded separately.

\section{A. Double-Zipper ZigZag}

In the original ZigZag [24], the decoupling process starts from one side (e.g., left to right) through the received overlapped packets. While in the double-zipper ZigZag, in order to accumulate less noise, the decoupling is performed from both sides towards the middle of the overlapped packets. We consider two cases $L_{2}>L_{1} \geq 0$, where $L_{t}:=L_{t, 2}-L_{t, 1}$, and $L_{2}>0 \geq L_{1}$. Note that relabeling the time-intervals relabels the delay differences $L_{1}$ and $L_{2}$ as well, while relabeling the users changes the signs of the delay differences. Clearly, by considering the case of $L_{2}>L_{1} \geq 0$, the cases of $L_{1}>L_{2} \geq 0$ (user relabeling), $L_{2}<L_{1} \leq 0$ (time-interval relabeling), and $L_{1}<L_{2} \leq 0$ (user and time-interval relabeling) are covered as well. Also, the case of $L_{2}>0 \geq L_{1}$ covers the cases of $L_{1}>0 \geq L_{2}, L_{2}<0 \leq L_{1}$, and $L_{1}<0 \leq L_{2}$.

Assuming $L_{2}>L_{1} \geq 0$, the double-zipper ZigZag proceeds as follows: in the first decoupling step, using the received signals in the second time-interval, the first $L_{2}$ symbols of the first user and the last $L_{2}$ symbols of the second user are observed without interference. Next, $L_{2}-L_{1}$ symbols of each user are removed of interference in each step using the observations of the previous step. The process continues in the same manner until both packets are interference free.

Let us describe the process more explicitly via an example with perfect CSI at the receiver. Decoupling steps of the original and double-zipper ZigZag are depicted in Fig. 3 and Fig. 4. respectively, for the case with $N=5$ and $L=\left[\begin{array}{ll}0 & 1 \\ 0 & 3\end{array}\right]$.

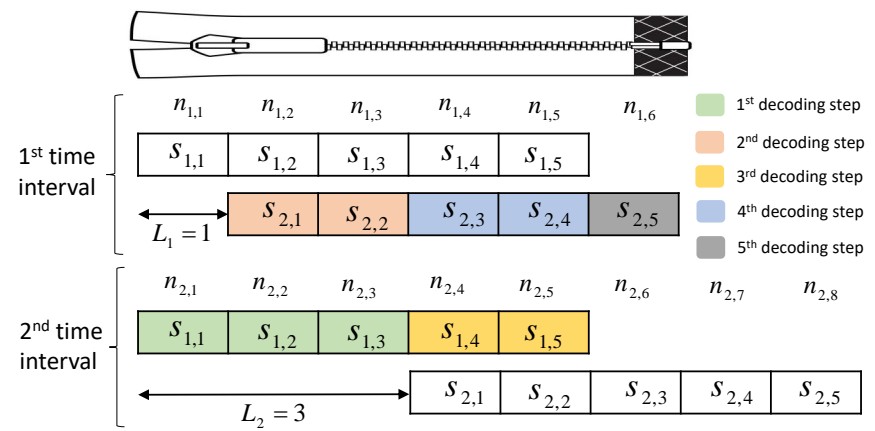

Fig. 3: Original ZigZag process for $N=5, L_{1}=1$ and $L_{2}=3$ $\left(L_{2}>L_{1} \geq 0\right)$.

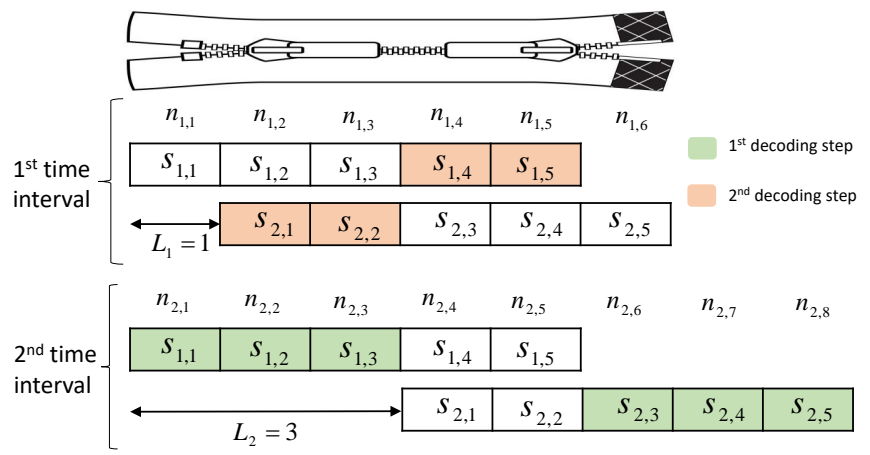

Fig. 4: Double-zipper ZigZag process for $N=5, L_{1}=1$ and $L_{2}=3\left(L_{2}>L_{1} \geq 0\right)$.

Based on Fig. 4, in the first step of the decoupling process, three interference free symbols of each user are

$$
\begin{aligned}
& \hat{s}_{1,1}=\frac{1}{h_{1}} \hat{y}_{1}=\frac{1}{h_{1}} y_{2,1}=s_{1,1}+\frac{1}{h_{1}} n_{2,1}, \\
& \hat{s}_{1,2}=\frac{1}{h_{1}} \hat{y}_{2}=\frac{1}{h_{1}} y_{2,2}=s_{1,2}+\frac{1}{h_{1}} n_{2,2}, \\
& \hat{s}_{1,3}=\frac{1}{h_{1}} \hat{y}_{3}=\frac{1}{h_{1}} y_{2,3}=s_{1,3}+\frac{1}{h_{1}} n_{2,3}, \\
& \hat{s}_{2,3}=\frac{1}{h_{2}} \hat{y}_{8}=\frac{1}{h_{2}} y_{2,6}=s_{2,3}+\frac{1}{h_{2}} n_{2,6}, \\
& \hat{s}_{2,4}=\frac{1}{h_{2}} \hat{y}_{9}=\frac{1}{h_{2}} y_{2,7}=s_{2,4}+\frac{1}{h_{2}} n_{2,7}, \\
& \hat{s}_{2,5}=\frac{1}{h_{2}} \hat{y}_{10}=\frac{1}{h_{2}} y_{2,8}=s_{2,5}+\frac{1}{h_{2}} n_{2,8},
\end{aligned}
$$

where $\hat{s}_{k, j}$ is the $j$-th decoupled (recovered) symbol of the $k$-th user and $\hat{y}_{i}$ is the $i$-th element of $\hat{\mathbf{y}}$. Also, using (14), the remaining four symbols are freed of interference in the second step, resulting in

$$
\begin{aligned}
& \hat{s}_{2,1}=\frac{1}{h_{2}} \hat{y}_{6}=\frac{1}{h_{2}}\left(y_{1,2}-h_{1} \hat{s}_{1,2}\right)=s_{2,1}+\frac{1}{h_{2}}\left(n_{1,2}-n_{2,2}\right), \\
& \hat{s}_{2,2}=\frac{1}{h_{2}} \hat{y}_{7}=\frac{1}{h_{2}}\left(y_{1,3}-h_{1} \hat{s}_{1,3}\right)=s_{2,2}+\frac{1}{h_{2}}\left(n_{1,3}-n_{2,3}\right), \\
& \hat{s}_{1,4}=\frac{1}{h_{1}} \hat{y}_{4}=\frac{1}{h_{1}}\left(y_{1,4}-h_{2} \hat{s}_{2,3}\right)=s_{1,4}+\frac{1}{h_{1}}\left(n_{1,4}-n_{2,6}\right), \\
& \hat{s}_{1,5}=\frac{1}{h_{1}} \hat{y}_{5}=\frac{1}{h_{1}}\left(y_{1,5}-h_{2} \hat{s}_{2,4}\right)=s_{1,5}+\frac{1}{h_{1}}\left(n_{1,5}-n_{2,7}\right) .
\end{aligned}
$$

Using these two sets of equations, the matrices in (2) are obtained as

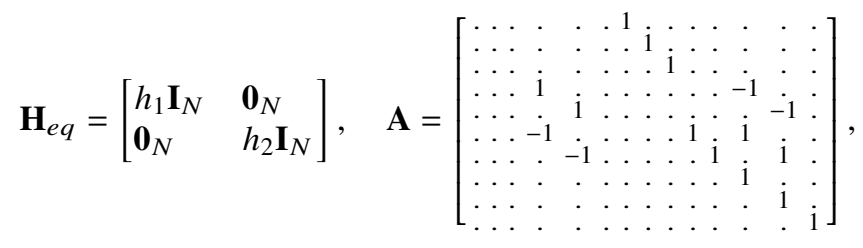

where $\mathbf{I}_{N}$ and $\mathbf{0}_{N}$ are the $N \times N$ identity and all-zero matrices, respectively, and the 0's in the matrix $\mathbf{A}$ are denoted by dots for better visibility. 


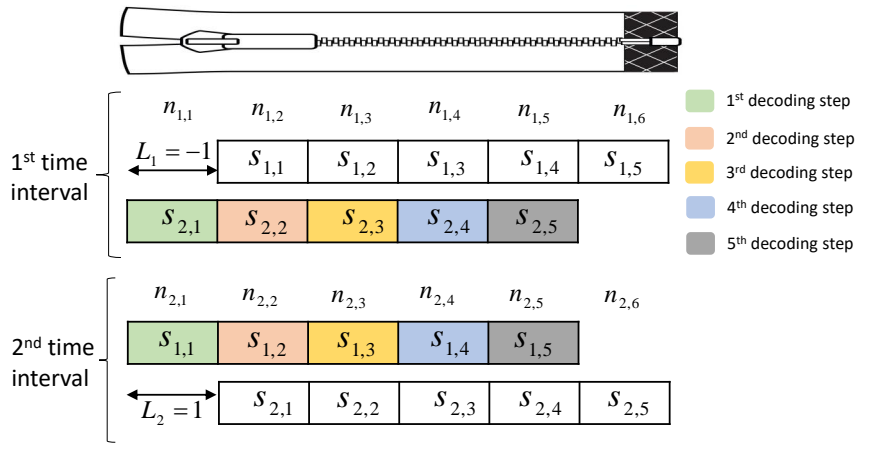

Fig. 5: Original ZigZag process for $N=5, L_{1}=-1$ and $L_{2}=1$ $\left(L_{2}>0 \geq L_{1}\right)$.

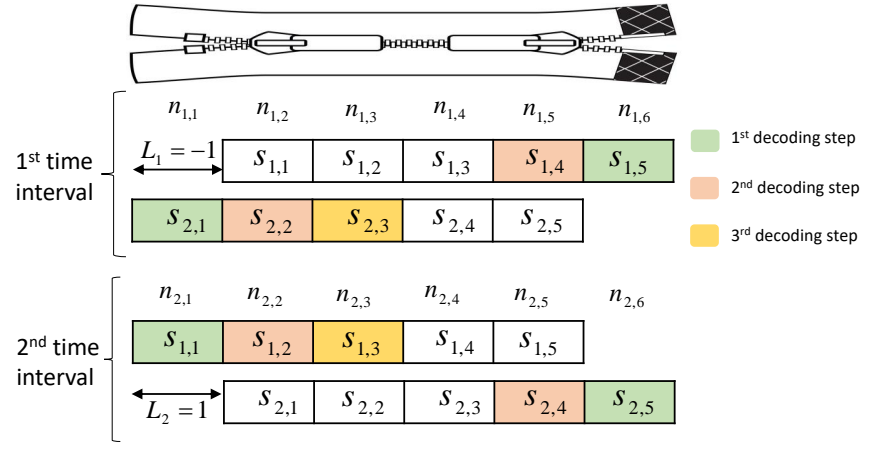

Fig. 6: Double-zipper ZigZag process for $N=5, L_{1}=-1$ and $L_{2}=1\left(L_{2}>0 \geq L_{1}\right)$.

The procedure for the double-zipper ZigZag is similar for the case of $L_{2}>0 \geq L_{1}$. In the first step, the first $L_{1}$ symbols of the second user and the last $L_{1}$ symbols of the first user are recovered. In the second time-interval, the first $L_{2}$ symbols of the first user and the last $L_{2}$ symbols of the second user are recovered as well. In the subsequent steps, $2\left(L_{2}-L_{1}\right)$ symbols are recovered in each step in a similar manner as in the case of $L_{2}>L_{1} \geq 0$. As examples, decoupling processes of the original and double-zipper ZigZag are depicted in Fig. 5 and Fig. 6 respectively, with $N=5$ and $L=\left[\begin{array}{ll}1 & 0 \\ 0 & 1\end{array}\right]$.

Note that since the users' packets decouple completely in both original and double-zipper ZigZag processes, their equivalent channel matrices are always equal to the identity matrix. Also, since the symbols are decoupled using SIC by simple additions and subtractions, the noise coefficient matrix is a ternary matrix of 0's, -1 's and 1's in ZigZag algorithms.

\section{B. Noise Covariance Matrix}

We first review the case of $L_{2}>L_{1} \geq 0$. In the first step of the original ZigZag process, $L_{2}$ symbols are available free of interference with only one input noise term. Next, in each middle step, $L_{2}-L_{1}$ symbols are obtained (with the exception of $r_{1}:=\left(N-L_{2}\right)$ mod $\left(L_{2}-L_{1}\right)$ symbols in third-to-last and second-to-last steps), accumulating an additional input noise term in each step. Finally, $L_{1}$ symbols are obtained in the last step with one input noise term. The process is completed after $\left\lceil\frac{N-L_{2}}{L_{2}-L_{1}}\right\rceil+\left\lceil\frac{N-L_{1}}{L_{2}-L_{1}}\right\rceil+2=2\left\lceil\frac{N-L_{2}}{L_{2}-L_{1}}\right\rceil+3$ steps. On the other hand, the double-zipper ZigZag process has $\left[\frac{N-L_{2}}{L_{2}-L_{1}}\right]+1$ steps, which translates to almost half of that of the original ZigZag for large $N$, leading to less noise accumulation. To be exact, $2 L_{2}$ symbols are available in the first step with one input noise term. Next, $2\left(L_{2}-L_{1}\right)$ symbols are obtained in each step $\left(2 r_{1}\right.$ symbols in the last step), accumulating an additional input noise term in each step.

The process is similar for the case of $L_{2}>0 \geq L_{1} . L_{2}-L_{1}$ symbols are available free of interference in the first step of the original ZigZag process, with only one input noise term. Next, $L_{2}-L_{1}$ symbols are obtained (with the exception of $r_{2}:=2 N \bmod \left(L_{2}-L_{1}\right)$ symbols in the last step) in each subsequent step, accumulating an additional input noise term for each. The process is completed after $\left[\frac{2 N}{L_{2}-L_{1}}\right]$ steps. On the other hand, the double-zipper ZigZag process has $\left\lceil\frac{N}{L_{2}-L_{1}}\right]$ steps, which translates to almost half of that of the original ZigZag, leading to less noise accumulation. To be exact, $2\left(L_{2}-L_{1}\right)$ symbols are available in its first step with one input noise term. Next, $2\left(L_{2}-L_{1}\right)$ symbols are obtained in each step $\left(r_{3}:=2 N \bmod 2\left(L_{2}-L_{1}\right)\right.$ symbols in the last step), accumulating an additional input noise term for each. Summing up, we obtain the following lemma.

Lemma 1. For the case of $L_{2}>L_{1} \geq 0$ : In the original ZigZag process, the diagonal of the noise covariance matrix $\mathbf{R}_{n}$ has $L_{1}+L_{2}$ elements equal to $\sigma_{n}^{2}, 2 M_{1}+1$ groups of $L_{2}-L_{1}$ elements each with value $k \sigma_{n}^{2}, k=2, \ldots, 2 M_{1}+2, r_{1}$ elements equal to $\left(2 M_{1}+3\right) \sigma_{n}^{2}$, and $r_{1}$ elements equal to $\left(2 M_{1}+4\right) \sigma_{n}^{2}$, where $M_{1}:=\left\lfloor\frac{N-L_{2}}{L_{2}-L_{1}}\right\rfloor$. While in the double-zipper ZigZag process, the diagonal of the noise covariance matrix $\mathbf{R}_{n}$ has $2 L_{2}$ elements equal to $\sigma_{n}^{2}, M_{1}$ groups of $2\left(L_{2}-L_{1}\right)$ elements each with value $k \sigma_{n}^{2}, k=2, \ldots, M_{1}+1$, and $2 r_{1}$ elements equal to $\left(M_{1}+2\right) \sigma_{n}^{2}$.

For the case of $L_{2}>0 \geq L_{1}$ : In the original ZigZag process, the diagonal of the noise covariance matrix $\mathbf{R}_{n}$ has $M_{2}$ groups of $L_{2}-L_{1}$ elements each with value $k \sigma_{n}^{2}, k=1, \ldots, M_{2}$, and $r_{2}$ elements equal to $\left(M_{2}+1\right) \sigma_{n}^{2}$, where $M_{2}:=\left\lfloor\frac{2 N}{L_{2}-L_{1}} \mid\right.$. While in the double-zipper ZigZag process, the diagonal of the noise covariance matrix $\mathbf{R}_{n}$ has $M_{3}$ groups of $2\left(L_{2}-L_{1}\right)$ elements each with value $k \sigma_{n}^{2}, k=1, \ldots, M_{3}$, and $r_{3}$ elements equal to $\left(M_{3}+1\right) \sigma_{n}^{2}$, where $M_{3}:=\left\lfloor\frac{N}{L_{2}-L_{1}}\right\rfloor$.

\section{Bounds on the Sum-Rate}

1) Unit Channel Gains With Perfect CSI: The following lemma proves that if we replace the correlated noise terms with independent terms of the same variances, the mutual information will not increase if the codebooks are constructed using independent elements.

Lemma 2. Let $Y^{N}=X^{N}+Z^{N}$ with $X^{N}=\left[X_{1}, X_{2}, \ldots, X_{N}\right]$ and $Z^{N}=\left[Z_{1}, Z_{2}, \ldots, Z_{N}\right]$. Assume that $X_{i}^{\prime}$ 's, and the vectors $X^{N}$ and $Z^{N}$ are independent; however, $Z_{i}$ 's may be correlated. Then, $I\left(X^{N} ; Y^{N}\right) \geq \sum_{i=1}^{N} I\left(X_{i} ; Y_{i}\right)$.

Proof: We first expand the mutual information term 


$$
I\left(X^{N} ; Y^{N}\right) \text { as }
$$

$$
\begin{aligned}
I\left(X^{N} ; Y^{N}\right) & =\sum_{i=1}^{N} I\left(X^{N} ; Y_{i} \mid Y^{i-1}\right) \\
& =\sum_{j=1}^{N} \sum_{i=1}^{N} I\left(X_{j} ; Y_{i} \mid X^{j-1}, Y^{i-1}\right) .
\end{aligned}
$$

Since mutual information is non-negative, keeping only the terms with $j=i$, we have

$$
I\left(X^{N} ; Y^{N}\right) \geq \sum_{i=1}^{N} I\left(X_{i} ; Y_{i} \mid X^{i-1}, Y^{i-1}\right) .
$$

Since $X_{i}$ is independent of $X^{i-1}$ and $Y^{i-1}$ (from the convexity of mutual information $I(U ; V)$ in $p(v \mid u)$ for fixed $p(u)$ [see [36], p. 25]), $I\left(X_{i} ; Y_{i} \mid X^{i-1}, Y^{i-1}\right) \geq I\left(X_{i} ; Y_{i}\right)$, and the claim follows.

Let the first and the second delay differences be $L_{1}=\beta_{1} N$ and $L_{2}=\beta_{2} N$, respectively.

Theorem 1. Let $R_{D Z}$ and $R_{O Z}$ be the achievable sum-rate per channel use of the double-zipper and the original ZigZag algorithms, respectively. Then, $R_{D Z}$ and $R_{O Z}$ can be lower bounded as follows.

For the case of $L_{2}>L_{1} \geq 0$ :

$$
\begin{aligned}
R_{D Z} \geq & \beta_{2} \log (1+\rho)+\frac{r_{1}}{N} \log \left(1+\frac{\rho}{M_{1}+2}\right) \\
& +\left(\beta_{2}-\beta_{1}\right) \sum_{k=1}^{M_{1}} \log \left(1+\frac{\rho}{k+1}\right),
\end{aligned}
$$

and

$$
\begin{aligned}
R_{O Z} \geq & \frac{\beta_{1}+\beta_{2}}{2} \log (1+\rho)+\frac{\beta_{2}-\beta_{1}}{2} \sum_{k=1}^{1+2 M_{1}} \log \left(1+\frac{\rho}{k+1}\right) \\
& +\frac{r_{1}}{2 N}\left(\log \left(1+\frac{\rho}{2 M_{1}+3}\right)+\log \left(1+\frac{\rho}{2 M_{1}+4}\right)\right), \quad \text { (19) }
\end{aligned}
$$

and for the case of $L_{2}>0 \geq L_{1}$ :

$$
R_{D Z} \geq \frac{r_{3}}{2 N} \log \left(1+\frac{\rho}{M_{3}+1}\right)+\left(\beta_{2}-\beta_{1}\right) \sum_{k=1}^{M_{3}} \log \left(1+\frac{\rho}{k}\right),
$$

and

$$
R_{O Z} \geq \frac{r_{2}}{2 N} \log \left(1+\frac{\rho}{M_{2}+1}\right)+\frac{\beta_{2}-\beta_{1}}{2} \sum_{k=1}^{M_{2}} \log \left(1+\frac{\rho}{k}\right) .
$$

Proof: We know that $\mathbf{H}_{e q}=\mathbf{I}_{2 N}$ for unit gain channels in ZigZag algorithms. So, using (4) and Lemma 2, the general sum-rate of ZigZag algorithms $\left(R_{Z Z}\right)$ can be written as:

$$
\begin{aligned}
R_{Z Z} & \geq \frac{1}{2 N} \sum_{k=1}^{2} \log \left(\frac{\left|\mathbf{R}_{n, k}^{\prime}+\rho \mathbf{I}_{N}\right|}{\left|\mathbf{R}_{n, k}^{\prime}\right|}\right) \\
& =\frac{1}{2 N} \log \left(\frac{\left|\mathbf{R}_{n}^{\prime}+\rho \mathbf{I}_{2 N}\right|}{\left|\mathbf{R}_{n}^{\prime}\right|}\right),
\end{aligned}
$$

where $\mathbf{R}_{n}^{\prime}$ and $\mathbf{R}_{n, k}^{\prime}$ are diagonal matrices with the same diagonal elements as $\mathbf{R}_{n}$ and $\mathbf{R}_{n, k}$, respectively, and the equality is obtained due to the fact that $\mathbf{R}_{n}^{\prime}=\left[\begin{array}{cc}\mathbf{R}_{n, 1}^{\prime} & \mathbf{0}_{N} \\ \mathbf{0}_{N} & \mathbf{R}_{n, 2}^{\prime}\end{array}\right]$.
Next, using Lemma 1, for the double-zipper ZigZag when $L_{2}>L_{1} \geq 0$, we have

$$
\begin{aligned}
\left|\mathbf{R}_{n}^{\prime}\right| & =\underbrace{\left(\sigma_{n}^{2}\right)^{2 L_{2}}}_{\text {first step }} \underbrace{\left(\left(M_{1}+2\right) \sigma_{n}^{2}\right)^{2 r_{1}}}_{\text {last step }} \underbrace{\prod_{k=1}^{M_{1}}\left((k+1) \sigma_{n}^{2}\right)^{2\left(L_{2}-L_{1}\right)}}_{\text {middle steps }} \\
& =\left(\sigma_{n}^{2}\right)^{2 N}\left(M_{1}+2\right)^{2 r_{1}} \prod_{k=1}^{M_{1}}(k+1)^{2\left(L_{2}-L_{1}\right)} .
\end{aligned}
$$

Also, we have

$$
\begin{aligned}
& \left|\mathbf{R}_{n}^{\prime}+P \mathbf{I}\right| \\
& =\underbrace{\left(\sigma_{n}^{2}+P\right)^{2 L_{2}}}_{\text {first step }} \underbrace{\left(\left(M_{1}+2\right) \sigma_{n}^{2}+P\right)^{2 r_{1}}}_{\text {last step }} \underbrace{\prod_{k=1}^{M_{1}}\left((k+1) \sigma_{n}^{2}+P\right)^{2\left(L_{2}-L_{1}\right)}}_{\text {middle steps }} \\
& =\left(\sigma_{n}^{2}\right)^{2 N}(1+\rho)^{2 L_{2}}\left(\left(M_{1}+2\right)+\rho\right)^{2 r_{1}} \prod_{k=1}^{M_{1}}((k+1)+\rho)^{2\left(L_{2}-L_{1}\right)} .
\end{aligned}
$$

Substituting (23) and (24) into (22), gives (18), i.e., the lower bound on the achievable sum-rate of the double-zipper ZigZag process. In a similar manner, the lower bounds on the achievable sum-rate of the original ZigZag when $L_{2}>L_{1} \geq 0$ and the achievable sum-rates of the double-zipper and the original ZigZag processes when $L_{2}>0 \geq L_{1}$ are obtained as in (19), 20) and (21), respectively.

2) Non-Unit Channel Gains With Perfect CSI: We now consider non-unit channel gains, which are taken as constant during the two time-intervals. We first assume that the receiver knows all the channel gains perfectly. It is easy to show that, for both ZigZag algorithms, the equivalent channel matrix in (2) is diagonal with the first half of diagonal entries being $h_{1}$ and the second half being $h_{2}$. So, for both cases, the achievable sum-rate (4) can be further simplified as

$$
R_{Z Z}=\frac{1}{2 N} \sum_{k=1}^{2} \log \left(\left.|\rho| h_{k}\right|^{2}\left(\mathbf{A}_{k} \mathbf{A}_{k}^{\mathrm{T}}\right)^{-1}+\mathbf{I}_{N} \mid\right) .
$$

In the double-zipper ZigZag, half of the symbols (to be decoupled in each step) are decoupled using the first channel gain and the other half using the second one. Using this fact, similar to Theorem 11 we obtain a lower bound on the achievable sum-rate (per channel use) for $L_{2}>L_{1} \geq 0$ as

$$
\begin{aligned}
R_{D Z} \geq & \frac{\beta_{2}}{2}\left(\log \left(1+\rho\left|h_{1}\right|^{2}\right)+\log \left(1+\rho\left|h_{2}\right|^{2}\right)\right) \\
& +\frac{\beta_{2}-\beta_{1}}{2} \sum_{k=1}^{M_{1}}\left(\log \left(1+\frac{\rho\left|h_{1}\right|^{2}}{k+1}\right)+\log \left(1+\frac{\rho\left|h_{2}\right|^{2}}{k+1}\right)\right) \\
& +\frac{r_{1}}{2 N}\left(\log \left(1+\frac{\rho\left|h_{1}\right|^{2}}{M+2}\right)+\log \left(1+\frac{\rho\left|h_{2}\right|^{2}}{M+2}\right)\right) .
\end{aligned}
$$


On the other hand, in the original ZigZag process, the associated channel gain changes in each step, and we obtain

$$
\begin{aligned}
R_{O Z} \geq & \frac{\beta_{1}}{2}\left(\log \left(1+\rho\left|h_{1}\right|^{2}\right)+\log \left(1+\rho\left|h_{2}\right|^{2}\right)\right) \\
& +\frac{\beta_{2}-\beta_{1}}{2} \sum_{k=0}^{M_{1}}\left(\log \left(1+\frac{\rho\left|h_{1}\right|^{2}}{2 k+1}\right)+\log \left(1+\frac{\rho\left|h_{2}\right|^{2}}{2 k+2}\right)\right) \\
& +\frac{r_{1}}{2 N}\left(\log \left(1+\frac{\rho\left|h_{1}\right|^{2}}{2 M+3}\right)+\log \left(1+\frac{\rho\left|h_{2}\right|^{2}}{2 M+4}\right)\right) .
\end{aligned}
$$

Also, for the case of $L_{2}>0 \geq L_{1}$, we have

$$
\begin{aligned}
R_{D Z} \geq & \frac{\beta_{2}-\beta_{1}}{2} \sum_{k=1}^{M_{3}}\left(\log \left(1+\frac{\rho\left|h_{1}\right|^{2}}{k}\right)+\log \left(1+\frac{\rho\left|h_{2}\right|^{2}}{k}\right)\right) \\
& +\frac{r_{3}}{4 N}\left(\log \left(1+\frac{\rho\left|h_{1}\right|^{2}}{M_{3}+1}\right)+\log \left(1+\frac{\rho\left|h_{1}\right|^{2}}{M_{3}+1}\right)\right),
\end{aligned}
$$

and

$$
\begin{aligned}
R_{O Z} \geq & -\frac{\beta_{1}}{2} \sum_{\substack{k=1 \\
\text { odd }}}^{M_{2}} \log \left(1+\frac{\rho\left|h_{2}\right|^{2}}{k}\right)+\frac{\beta_{2}}{2} \sum_{\substack{k=2 \\
\text { even }}}^{M_{2}} \log \left(1+\frac{\rho\left|h_{2}\right|^{2}}{k}\right) \\
& +\frac{\beta_{2}}{2} \sum_{\substack{k=1 \\
\text { odd }}}^{M_{2}} \log \left(1+\frac{\rho\left|h_{1}\right|^{2}}{k}\right)-\frac{\beta_{1}}{2} \sum_{\substack{k=2 \\
\text { even }}}^{M_{2}} \log \left(1+\frac{\rho\left|h_{1}\right|^{2}}{k}\right) \\
& +\frac{r_{2,1}}{2 N} \log \left(1+\frac{\rho\left|h_{1}\right|^{2}}{M_{2}+1}\right)+\frac{r_{2,2}}{2 N} \log \left(1+\frac{\rho\left|h_{2}\right|^{2}}{M_{2}+1}\right), \text { (29) }
\end{aligned}
$$

where $r_{2,1}:=\left(N \bmod \left(L_{2}-L_{1}\right)\right) \bmod L 2$ and $r_{2,2} \quad:=$ $\left(N \bmod \left(L_{2}-L_{1}\right)\right) \bmod -L_{1}$. Note that $r_{2,1}+r_{2,2}=r_{2}$.

3) Non-Unit Channel Gains With Imperfect CSI: For the ZigZag algorithm with imperfect CSI, since the channel estimates are not used in the decoupling process, the postprocessing system model is the same as the perfect CSI case, i.e., $\overline{\mathbf{H}}_{e q}$ and $\tilde{\mathbf{H}}_{e q}$ are diagonal matrices consisting of the channel estimates and the channel estimation errors, respectively. So, using (10) and (11), the lower and upper bounds on the achievable sum-rate (per channel use) of ZigZag algorithms for single-user decoding in the presence of imperfect CSI are obtained as

$$
R_{Z Z} \geq \frac{1}{2 N} \sum_{k=1}^{2} \log \left(\left.|\rho| \bar{h}_{k}\right|^{2}\left(c \mathbf{I}_{N}+\mathbf{A}_{k} \mathbf{A}_{k}^{\mathrm{T}}\right)^{-1}+\mathbf{I}_{N} \mid\right),
$$

and

$$
R_{Z Z} \leq \frac{1}{2 N} \sum_{k=1}^{2} \log \left(\left|\left(c+\rho\left|\bar{h}_{k}\right|^{2}\right)\left(\mathbf{A}_{k} \mathbf{A}_{k}^{\mathrm{T}}\right)^{-1}+\mathbf{I}_{N}\right|\right)
$$

respectively, with equalities when perfect CSI is available (i.e., $c=0$ ), in which case (30) and (31) turn into 25.

Keeping only the diagonals of the noise covariance matrix and using the results in [35], we can extend the lower bounds
(26)-(29) to the case of imperfect CSI as follows. For $L_{2}>$ $L_{1} \geq 0$, we have

$$
\begin{aligned}
R_{D Z} \geq & \frac{\beta_{2}}{2}\left(\log \left(1+\frac{\rho\left|\bar{h}_{1}\right|^{2}}{c+1}\right)+\log \left(1+\frac{\rho\left|\bar{h}_{2}\right|^{2}}{c+1}\right)\right) \\
& +\frac{\beta_{2}-\beta_{1}}{2} \sum_{k=1}^{M}\left(\log \left(1+\frac{\rho\left|\bar{h}_{1}\right|^{2}}{k+c+1}\right)+\log \left(1+\frac{\rho\left|\bar{h}_{2}\right|^{2}}{k+c+1}\right)\right) \\
& +\frac{r}{2 N}\left(\log \left(1+\frac{\rho\left|\bar{h}_{1}\right|^{2}}{M+c+2}\right)+\log \left(1+\frac{\rho\left|\bar{h}_{2}\right|^{2}}{M+c+2}\right)\right)
\end{aligned}
$$

$$
\begin{aligned}
R_{O Z} \geq & \frac{\beta_{1}}{2}\left(\log \left(1+\frac{\rho\left|\bar{h}_{1}\right|^{2}}{c+1}\right)+\log \left(1+\frac{\rho\left|\bar{h}_{2}\right|^{2}}{c+1}\right)\right) \\
& +\frac{\beta_{2}-\beta_{1}}{2} \sum_{k=0}^{M}\left(\log \left(1+\frac{\rho\left|\bar{h}_{1}\right|^{2}}{2 k+c+1}\right)+\log \left(1+\frac{\rho\left|\bar{h}_{2}\right|^{2}}{2 k+c+2}\right)\right) \\
& +\frac{r}{2 N}\left(\log \left(1+\frac{\rho\left|\bar{h}_{1}\right|^{2}}{2 M+c+3}\right)+\log \left(1+\frac{\rho\left|\bar{h}_{2}\right|^{2}}{2 M+c+4}\right)\right) .
\end{aligned}
$$

Also, for the case of $L_{2}>0 \geq L_{1}$, we have

$$
\begin{aligned}
R_{D Z} \geq & \frac{\beta_{2}-\beta_{1}}{2} \sum_{k=1}^{M_{3}}\left(\log \left(1+\frac{\rho\left|h_{1}\right|^{2}}{k+c}\right)+\log \left(1+\frac{\rho\left|h_{2}\right|^{2}}{k+c}\right)\right) \\
& +\frac{r_{3}}{4 N}\left(\log \left(1+\frac{\rho\left|h_{1}\right|^{2}}{M_{3}+c+1}\right)+\log \left(1+\frac{\rho\left|h_{1}\right|^{2}}{M_{3}+c+1}\right)\right), \\
R_{O Z} \geq & -\frac{\beta_{1}}{2} \sum_{k=1}^{M_{2}} \log \left(1+\frac{\rho\left|h_{2}\right|^{2}}{k+c}\right)+\frac{\beta_{2}}{2} \sum_{\substack{k=2 \\
\text { even }}}^{M_{2}} \log \left(1+\frac{\rho\left|h_{2}\right|^{2}}{k+c}\right) \\
& +\frac{\beta_{2}}{2} \sum_{k=1}^{M_{2}} \log \left(1+\frac{\rho\left|h_{1}\right|^{2}}{k+c}\right)-\frac{\beta_{1}}{2} \sum_{\substack{k=2 \\
\text { even }}}^{M_{2}} \log \left(1+\frac{\rho\left|h_{1}\right|^{2}}{k+c}\right) \\
& +\frac{r_{2,1}}{2 N} \log \left(1+\frac{\rho\left|h_{1}\right|^{2}}{M_{2}+c+1}\right)+\frac{r_{2,2}}{2 N} \log \left(1+\frac{\rho\left|h_{2}\right|^{2}}{M_{2}+c+1}\right) .
\end{aligned}
$$

Note that when perfect CSI is available (i.e., $c=0$ ), 32)- 35 turn into [26)-29], respectively.

\section{Asymptotic Optimality}

In this section, we show that the ZigZag algorithms are asymptotically optimal. To this end, we prove that the gap between the lower bound on the achievable sum-rate of the ZigZag algorithms and the upper bound on the time-sharing sum-rate becomes a constant at high SNRs, hence the relative performance gap (normalized over the SNR) goes to zero. We also provide some upper bounds on the asymptotic performance gap.

Theorem 2. In a multiple access system with imperfect CSI, employing a decoupling algorithm that results in a postprocessing system model as in (8) with diagonal $\overline{\mathbf{H}}_{e q}$ and $\tilde{\mathbf{H}}_{e q}$ (such as ZigZag), followed by a single-user decoding algorithm is asymptotically optimal. That is, the gap between the lower bound on the achievable sum-rate and the upper 
bound on the time-sharing sum-rate is upper bounded in high SNRs by a constant, i.e.,

$$
C_{T S}^{h i g h-S N R}-R_{e q}^{h i g h-S N R} \leq \log (K)+\frac{1}{K N} \sum_{k=1}^{K N} \log \left(c+R_{n, j j}\right),
$$

where $R_{n, j j}$ is the $j$-th diagonal element of $\mathbf{A A}^{\mathrm{T}}$.

Proof: In the high SNR regime, the upper bound on the time-sharing sum-rate capacity (13) can be simplified to

$$
C_{T S}^{h i g h-S N R} \leq \log (\rho)+\log (K)+\frac{1}{K} \sum_{k=1}^{K} \log \left(\left|\bar{h}_{k}\right|^{2}\right) .
$$

Also, using the results in [35], the lower bound on the achievable sum-rate of the post-processing system model of (8) can be obtained as

$$
\begin{aligned}
R_{e q} & \geq \frac{1}{K N} \log \left(\left|\rho\left(c \mathbf{I}_{K N}+\mathbf{A} \mathbf{A}^{\mathrm{T}}\right)^{-1} \overline{\mathbf{H}}_{e q} \overline{\mathbf{H}}_{e q}^{\mathrm{H}}+\mathbf{I}_{K N}\right|\right) \\
& \geq \frac{1}{K N} \sum_{j=1}^{K N} \log \left(1+\frac{\rho\left|\bar{H}_{j, j}\right|^{2}}{c+R_{n, j j}}\right),
\end{aligned}
$$

where $\bar{H}_{j, j}$ and $R_{n, j j}$ are the $j$-th diagonal elements of $\overline{\mathbf{H}}_{e q}$ and $\mathbf{A} \mathbf{A}^{\mathrm{T}}$, respectively. In 38 the first and the second inequalities are obtained based on the results in [35] and Lemma 2, respectively. Note that the first inequality in (38) is only valid for multiuser decoding, while the second one is valid for the single-user decoding as well.

In the high SNR regime, the lower bound on the sum-rate (38) can be simplified to

$$
R_{e q}^{h i g h-S N R} \geq \log (\rho)+\frac{1}{K} \sum_{k=1}^{K} \log \left(\left|\bar{h}_{k}\right|^{2}\right)-\frac{1}{K N} \sum_{k=1}^{K N} \log \left(c+R_{n, j j}\right) .
$$

Eq. (36) is obtained using (37) and 239, which concludes the proof.

Needless to say, Theorem 2 holds for the case of perfect CSI as well by setting $c=0$. We can conclude from Theorem 2 that the performances of both original and double-zipper ZigZag algorithms approach that of optimal coordinated time-sharing based solutions asymptotically for arbitrary user delays.

Corollary 1. For the original and double-zipper ZigZag algorithms the following upper bounds on the asymptotic performance gap hold.

For $L_{2}>L_{1} \geq 0$ (and its equivalent cases):

$$
\begin{aligned}
& C_{T S}^{h i g h-S N R}-R_{D Z}^{h i g h-S N R} \leq 1+\beta_{2} \log (c+1)+\frac{r}{N} \log (M+c+2) \\
& +\left(\beta_{2}-\beta_{1}\right) \log \left(\frac{\Gamma(M+c+2)}{\Gamma(c+2)}\right), \\
& C_{T S}^{h i g h-S N R}-R_{O Z}^{h i g h-S N R} \leq 1+\beta_{1} \log (c+1)+(M+1)\left(\beta_{2}-\beta_{1}\right) \\
& +\frac{r}{2 N} \log ((2 M+c+3)(2 M+c+4)) \\
& +\frac{\beta_{2}-\beta_{1}}{2} \log \left(\frac{\Gamma(2 M+c+3)}{\Gamma(c+1)}\right),
\end{aligned}
$$

where $\Gamma(\cdot)$ is the gamma function.
For $L_{2}>0 \geq L_{1}$ (and its equivalent cases):

$$
\begin{aligned}
C_{T S}^{\text {high-SNR }}-R_{D Z}^{\text {high-SNR }} \leq & +\left(\beta_{2}-\beta_{1}\right) \log \left(\frac{\Gamma\left(M_{3}+c+1\right)}{\Gamma(c+1)}\right) \\
& +\frac{r_{3}}{2 N} \log \left(M_{3}+c+1\right), \quad(42) \\
C_{T S}^{\text {high-SNR }}-R_{O Z}^{\text {high-SNR }} \leq 1 & +\frac{\beta_{2}+\beta_{1}}{2} r_{M_{2}} \log \frac{\left|h_{2}\right|}{\left|h_{1}\right|}+\frac{r_{2,1}}{2 N} \log \left|h_{2}\right| \\
& +\frac{r_{2,2}}{2 N} \log \left|h_{1}\right|+\frac{r_{2}}{2 N} \log \left(M_{2}+c+1\right) \\
& +\frac{\beta_{2}-\beta_{1}}{2} \log \left(\frac{\Gamma\left(2\left\lfloor\frac{M_{2}}{2}\right\rfloor+c+1\right)}{\Gamma(c+1)}\right) \\
& +\frac{\beta_{2}-\beta_{1}}{2} r_{M_{2}} \log \left(M_{2}+c\right),
\end{aligned}
$$

where $r_{M_{2}}:=M_{2} \bmod 2$.

Proof: Eq. 407- 43 can readily be obtained using 36 and Lemma 1 after some calculus. In obtaining these upper bounds, we have used the fact that $\prod_{k=a}^{b}(k+c)=\frac{\Gamma(b+c+1)}{\Gamma(a+c)}$, $\beta_{2}+\frac{r}{N}+M_{1}\left(\beta_{2}+\beta_{1}\right)=1,2\left(L_{2}-L_{1}\right) M_{3}+r_{3}=2 N$, $\left(L_{2}-L_{1}\right) M_{2}+r_{2}=2 N$, and $2\left\lfloor\frac{M_{2}}{2}\right\rfloor+r_{M_{2}}=M_{2}$.

Note that another option for the two user case is first denoising the recovered symbols in each step, then subtracting them from the received signal in the next step. We do not discuss this approach in any detail since the main focus of this paper is to prove the asymptotic optimality of the ZigZag algorithms. In the case of more than two users, however, the combined de-noising becomes infeasible in general since there may be no individual symbols to begin the denoising process.

\section{Arbitrary Number of Users: Delay Domain APPROACH}

ZigZag algorithms utilized in the previous section cannot be readily extended to decouple the users' packets when there are arbitrary number of users. Therefore, in this section, we develop simple collision resolution algorithms utilizing the noisy received signals in a systematic manner in delay domain. We first introduce linear equalization in the delay domain in order to decouple the users' packets. We then show the connection between the delay domain approach and the ZigZag algorithms (for the case of two users) for further explanation.

Using (1), after equalization at the receiver, we have

$$
\begin{aligned}
\mathbf{y}^{\prime}(D) & =\mathbf{W}(D) \mathbf{y}(D) \\
& =\mathbf{W}(D) \mathbf{L}(D) \mathbf{H} \mathbf{s}(D)+\mathbf{W}(D) \mathbf{n}(D),
\end{aligned}
$$

where $\mathbf{W}(D)$ is a $K \times K$ delay-domain equalizing matrix intended to decouple the desired users' signals. After the equalization, the receiver discards the extra terms of $\mathbf{y}^{\prime}(D)$, e.g., keeping only the terms up to the power $N-1$ in $k$-th entry as the estimated packet of $k$-th user. It then applies single user decoding on each entry in order to decode the users' packets.

By omitting the extra terms in the polynomials (terms with higher powers than $N-1$ ), (44) can easily be rewritten as the post-processing system model in (2). Hence, the bounds for 
single-user decoding given in (10) and (11) can be employed for analysis, where $\mathbf{H}_{e q}$ and $\mathbf{A}$ can be obtained by rewriting $\mathbf{W}(D) \mathbf{L}(D) \mathbf{H s}(D)$ and $\mathbf{W}(D) \mathbf{n}(D)$ as $\mathbf{H}_{e q} \mathbf{s}$ and $\mathbf{A n}$, respectively.

\section{A. Delay-Domain Zero Forcing}

In this subsection, we propose delay-domain zero forcing (DD-ZF) to extend decoupling of the users' packets to the case of arbitrary number of users. We first consider the case that the number of time-intervals is equal to the number of users, i.e., $T=K$. Assuming that $\mathbf{L}(D)$ is invertible, utilizing the zero forcing concept, the equalizing matrix in the delay domain is given by

$$
\mathbf{W}_{Z F}(D)=\mathbf{L}^{-1}(D) .
$$

Note that $\mathbf{W}_{Z F}(D)$ only needs to contain the clean signals (in polynomial form) up to a certain power of $D$. Also, in order for the delay-domain equalizing matrix to be implementable, it needs to have finite-order polynomial entries. The following lemma provides a way to reach this end.

Lemma 3. The inverse of matrix $\mathbf{X}(D)$ in the delay domain up to the power $N-1, \operatorname{inv}_{N-1}(\mathbf{X}(D))$, assuming that $\mathbf{H}(D)$ has a non-zero determinant, can be expressed as

$$
\operatorname{inv}_{N-1}(\mathbf{X}(D))=\frac{D^{-\gamma}}{\lambda} \sum_{j=0}^{N+\gamma-1} f^{j}(D) \operatorname{adj}(\mathbf{X}(\mathrm{D})),
$$

where $\operatorname{adj}(\cdot)$ denotes the adjugate operator and $\lambda D^{\gamma}$ is the term of $|\mathbf{H}(D)|$ with the least degree.

Proof: We first decompose $\mathbf{X}^{-1}(D)$ as

$$
\mathbf{X}^{-1}(D)=\frac{1}{|\mathbf{X}(D)|} \operatorname{adj}(\mathbf{X}(\mathrm{D})) \text {. }
$$

Since $\mathbf{X}(D)$ is a polynomial matrix, both $|\mathbf{X}(D)|$ and $\operatorname{adj}(\mathbf{X}(D))$ become polynomial matrices as well. Next, using Taylor series expansion, we write $\frac{1}{|\mathbf{X}(D)|}$ as a polynomial as follows

$$
\begin{aligned}
\frac{1}{|\mathbf{X}(D)|} & =\frac{1}{\lambda D^{\gamma}(1-f(D))} \\
& =\frac{D^{-\gamma}}{\lambda} \sum_{j=0}^{\infty} f^{j}(D) \\
& \stackrel{N-1}{\equiv} \frac{D^{-\gamma}}{\lambda} \sum_{j=0}^{N+\gamma-1} f^{j}(D),
\end{aligned}
$$

where $\stackrel{p}{=}$ denotes equivalence up to the power $p$. Since the packet length is $N$, we only need terms of up to the power $N-1$. Since the term with the least degree in $f(D)$ has at least a power of one, it is enough to have a summation up to $j=N+\gamma-1$, which concludes the proof.

Lemma 3 guarantees that $\operatorname{inv}_{N-1}(\mathbf{X}(D)) \mathbf{X}(D)$ is the same as the identity matrix up to the power $N-1$ (first $N$ terms). Therefore, using Lemma 3, the equalizing matrix for DD-ZF becomes

$$
\mathbf{W}_{D D-Z F}(D)=\operatorname{inv}_{N-1}(\mathbf{L}(D)) .
$$

Extension of the result to the case of $T \geq K$ is straightforward. In this case, using Lemma 3, we have

$$
\mathbf{W}_{D D-Z F}(D)=\operatorname{inv}_{N-1}\left(\mathbf{L}^{\mathrm{T}}(D) \mathbf{L}(D)\right) \mathbf{L}^{\mathrm{T}}(D) .
$$

Since $\mathbf{W}_{D D-Z F}(D) \mathbf{L}(D)$ is the same as the identity matrix up to power $N-1$, the DD-ZF is able to completely decouple the users' packets. So, similar to ZigZag algorithms, the equivalent channel matrix in this case is equal to a diagonal matrix with the first $N$ diagonal elements equal to $h_{1}$, the second set of $N$ diagonal elements equal to $h_{2}$, and so on.

For the two-user case, although the DD-ZF results in an equivalent channel matrix with the ZigZag algorithms, the resultant noise coefficient matrix is different, and it is obtained by rewriting $\mathbf{L}^{-1}(D) \mathbf{n}(D)$ as An. Accordingly, for the DD-ZF based decoupling, the achievable sum-rate (4) becomes

$$
R_{D D-Z F}=\frac{1}{T N} \sum_{k=1}^{K} \log \left(\left.|\rho| h_{k}\right|^{2}\left(\mathbf{A}_{k} \mathbf{A}_{k}^{\mathrm{T}}\right)^{-1}+\mathbf{I}_{N} \mid\right) .
$$

1) Connection Between the DD-ZF and ZigZag: In order to make the connection between the ZigZag algorithms and the delay-domain approach more lucid, we present an example with the same set of parameters as the first example in Section III, namely, $K=2, N=5, L_{1}=1$ and $L_{2}=3$. Using (49), the DD-ZF equalizing matrix is obtained as

$$
\mathbf{W}_{D D-Z F}(D)=\left[\begin{array}{cc}
-D^{2}-D^{4} & 1+D^{2}+D^{4} \\
D^{-1}+D+D^{3} & -D^{-1}-D-D^{3}
\end{array}\right] .
$$

By multiplying this matrix by vector of received signals $\mathbf{y}(D)$ and discarding the terms with orders higher than $N-1$ (taking first $N$ terms), we obtain an estimate of transmitted signals. For example, the first estimated signal $y_{1}^{\prime}(D)$ is obtained as

$$
y_{1}^{\prime}(D)=\left(-D^{2}-D^{4}\right) y_{1}(D)+\left(1+D^{2}+D^{4}\right) y_{2}(D),
$$

which in the time-domain can be written as

$$
y_{1}^{\prime}(t)=-y_{1}(t-2)-y_{1}(t-4)+y_{2}(t)+y_{2}(t-2)+y_{2}(t-4) .
$$

Finally, the first $N$ time slots of $y_{1}^{\prime}(t)$ gives us an estimate of the first user's transmitted packet. This time-domain interpretation and process is depicted in Fig. (7). Note that we have ignored noise in this figure for simplicity of depiction.

\section{B. Regularized $D D-Z F$}

Completely decoupling the users' packets is not necessarily the best strategy even with single user decoding treating interference as noise. With this motivation, using Lemma 3. we propose a regularized DD-ZF equalizer as follows

$$
\mathbf{W}_{R-D D-Z F}(D)=\operatorname{inv}_{N-1}\left(\mathbf{L}^{\mathrm{T}}(D) \mathbf{L}(D)+\alpha \mathbf{I}_{K}\right) \mathbf{L}^{\mathrm{T}}(D),
$$

where $\alpha$ is the regularization coefficient. Intuitively, a smaller value of $\alpha$ should be selected for higher SNR values. A good option could be to select it inversely proportional to the SNR. 


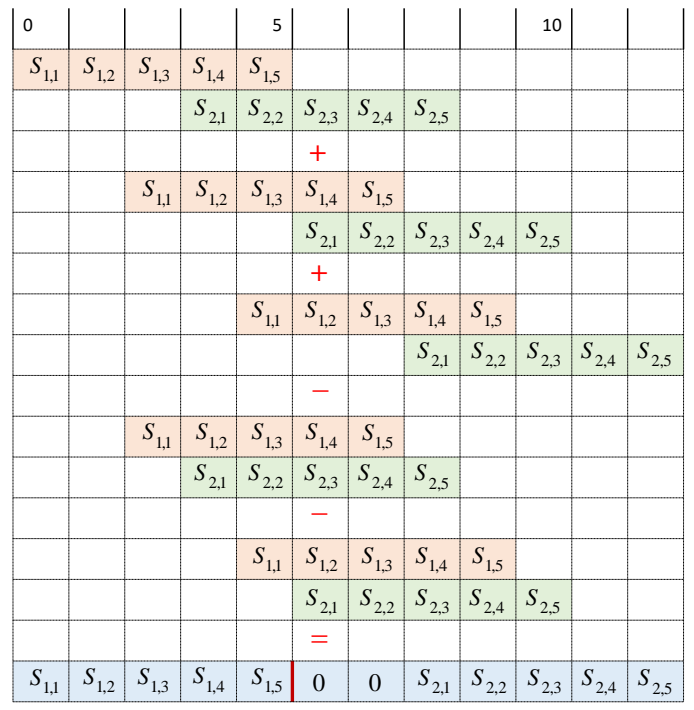

Fig. 7: Interpretation of DD-ZF in time domain for the twouser case with $N=5, L_{1}=1$ and $L_{2}=3$.

\section{Imperfect CSI Case}

In the delay domain with imperfect CSI, after equalization at the receiver, we have

$$
\mathbf{y}^{\prime}(D)=\mathbf{W}(D) \mathbf{L}(D)(\overline{\mathbf{H}}+\tilde{\mathbf{H}}) \mathbf{s}(D)+\mathbf{W}(D) \mathbf{n}(D),
$$

where $\overline{\mathbf{H}}$ and $\tilde{\mathbf{H}}$ are $K \times K$ diagonal matrices with the $k$-th diagonal entries equal to $\bar{h}_{k}$ and $\tilde{h}_{k}$, respectively. In this case, the bounds for single-user decoding are given in 10 and 11), where $\overline{\mathbf{H}}_{e q}, \tilde{\mathbf{H}}_{e q}$ and An can be obtained by rewriting $\overline{\mathbf{W}}(D) \mathbf{L}(D) \overline{\mathbf{H}} \mathbf{s}(D), \mathbf{W}(D) \mathbf{L}(D) \tilde{\mathbf{H}} \mathbf{s}(D)$ and $\mathbf{W}(D) \mathbf{n}(D)$ as $\overline{\mathbf{H}}_{e q} \mathbf{s}, \tilde{\mathbf{H}}_{e q} \mathbf{s}$ and An, respectively. Note that since the DD-ZF decouples the users' packets (i.e., cancels out the interference) resulting in diagonal channel matrices, according to Theorem 2 it is asymptotically optimal, even in the presence of imperfect CSI.

For the DD-ZF, imperfect CSI achievable rate bounds become

$$
\begin{aligned}
& R_{D D-Z F} \geq \frac{1}{T N} \sum_{k=1}^{K} \log \left(\left.|\rho| \bar{h}_{k}\right|^{2}\left(c \mathbf{I}_{N}+\mathbf{A}_{k} \mathbf{A}_{k}^{\mathrm{T}}\right)^{-1}+\mathbf{I}_{N} \mid\right), \\
& R_{D D-Z F} \leq \frac{1}{T N} \sum_{k=1}^{K} \log \left(\left|\left(c+\rho\left|\bar{h}_{k}\right|^{2}\right)\left(\mathbf{A}_{k} \mathbf{A}_{k}^{\mathrm{T}}\right)^{-1}+\mathbf{I}_{N}\right|\right),
\end{aligned}
$$

with equalities when perfect CSI is available (i.e., when $c=0$ ), in which case (57) and (58) turn into (51).

\section{Computational Complexity}

A naive way to apply the equalizers is to convert (1) into time domain as

$$
\mathbf{y}=\mathbf{H}_{T D} \mathbf{S}+\mathbf{I}_{T\left(N+L_{\max }\right)} \mathbf{n},
$$

where $\mathbf{y}=\left[\mathbf{y}_{1}^{\mathrm{T}}, \ldots, \mathbf{y}_{T}^{\mathrm{T}}\right]^{\mathrm{T}}$ with $\mathbf{y}_{t}=\left[y_{t, 1}, \ldots, y_{t, N+L_{\max }}\right]^{\mathrm{T}}$ and $\mathbf{H}_{T D}$ is a $K\left(N+L_{\text {max }}\right) \times K N$ block matrix with $\left(N+L_{\text {max }}\right) \times N$

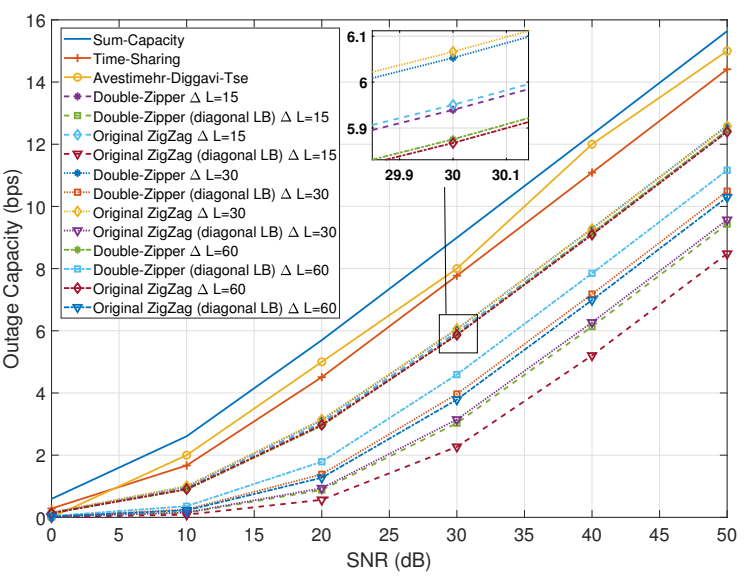

Fig. 8: Outage capacity of ZigZag with perfect CSI.

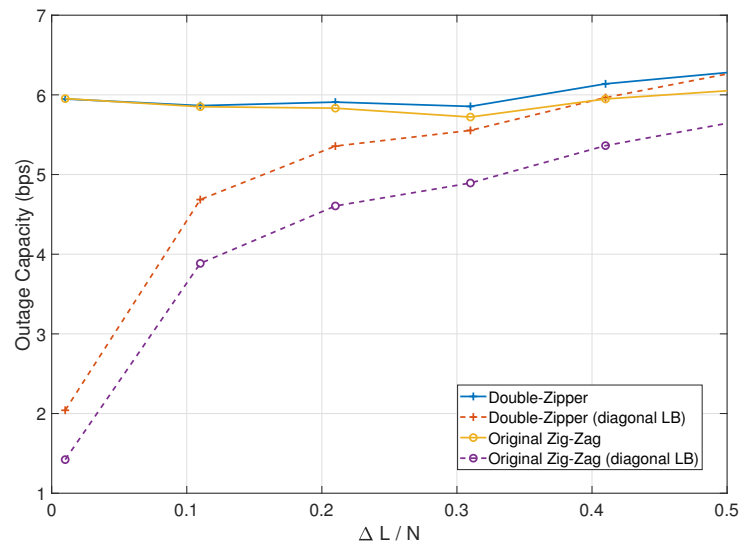

Fig. 9: Outage capacity of ZigZag with perfect CSI vs normalized delay difference.

blocks of the form $\mathbf{H}_{T D}^{t, k}=\left[\mathbf{0}_{N \times L_{t, k}}, h_{k} \mathbf{I}_{N}, \mathbf{0}_{N \times\left(L_{\max }-L_{t, k}\right)}\right]^{\mathrm{T}}$, and then apply conventional time-domain equalizers such as zero forcing. However, this will lead to a computational complexity of $O\left(K N T^{2}\left(N+L_{\max }\right)^{2}\right)$, which is much higher than the proposed delay-domain equalizers with a complexity of $O\left(K T^{2}\right)$.

\section{Numerical Results}

We compare the performance of the proposed scheme with that of (coordinated) time-sharing in both perfect and imperfect CSI scenarios. We assume Rayleigh fading with unit variance channel gains, which are constant during $T$ timeintervals. We have set the default values of outage probability to $10 \%, T=K, N=600, c=1$ (i.e., $\sigma_{h}^{2}=\frac{1}{\rho}$ ), $\mathbf{L}=\mathbf{I}_{K}$ for delay-domain algorithms, and $\beta_{1}=0$ and $\beta_{2}=0.05\left(L_{2}=30\right)$ for the ZigZag algorithms.

The outage capacity with perfect CSI is plotted versus the SNR in Fig. 8 for different delay values $\left(\Delta L:=L_{2}-L_{1}\right)$. It can be seen that the original ZigZag performs slightly better than the double-zipper ZigZag. This can be due to the observation that although the double-zipper algorithm accumulates less noise, the higher levels of noise correlation (off-diagonal elements in the noise correlation matrix $\mathbf{R}_{\mathbf{n}}$ ) in the original ZigZag work in its favor. Furthermore, ZigZag 


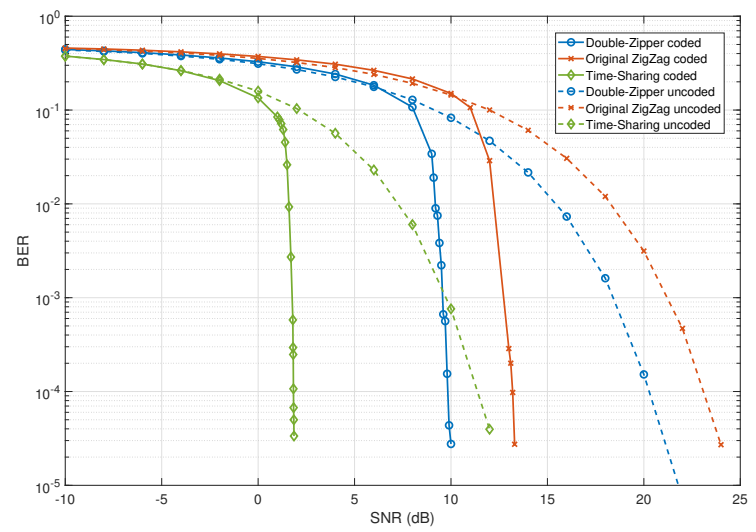

Fig. 10: BER performance of finite blocklength ZigZag with perfect CSI.

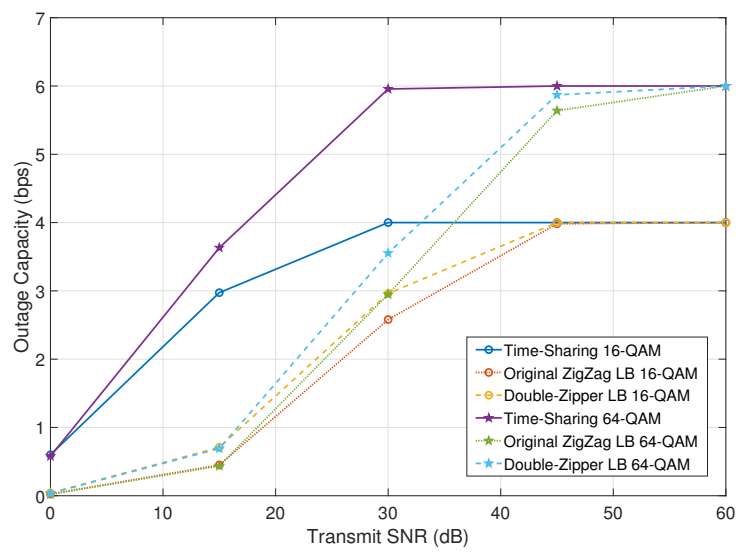

Fig. 11: Finite-alphabet achievable rate of ZigZag with perfect CSI.

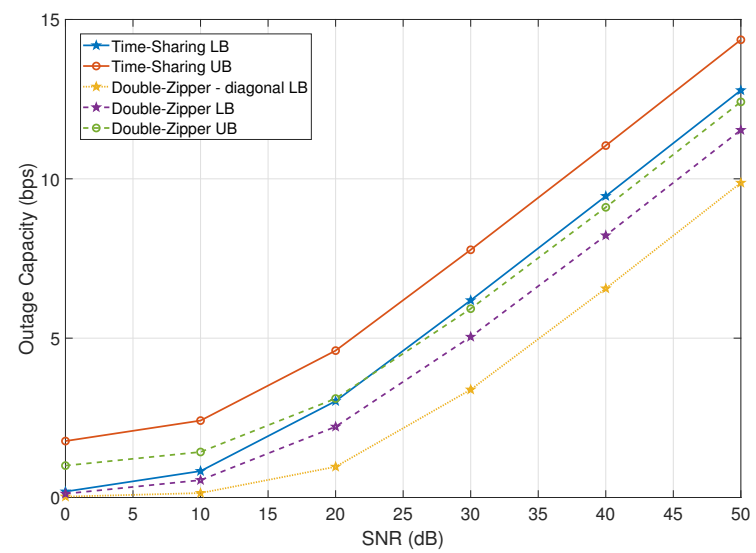

Fig. 12: Outage capacity with imperfect CSI.

even outperforms the result in [32] for some SNR values (see (7)). Constant capacity gap between the ZigZag performance and the sum capacity at high SNRs is also clear.

The outage capacity with perfect CSI is plotted versus the normalized delay difference in Fig. 9 It is observed that the performance of both ZigZag algorithms are almost unchanged when the delay difference increases, and the proposed lower bounds become tighter.

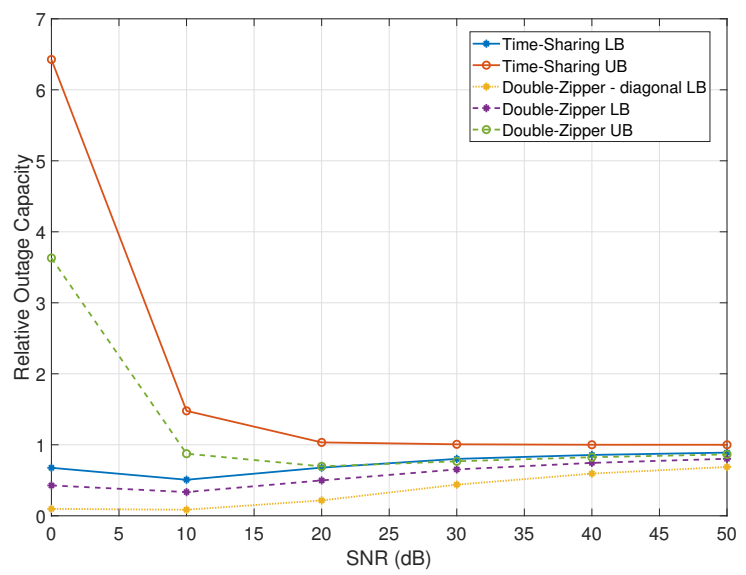

Fig. 13: Relative outage capacity of ZigZag with imperfect CSI.

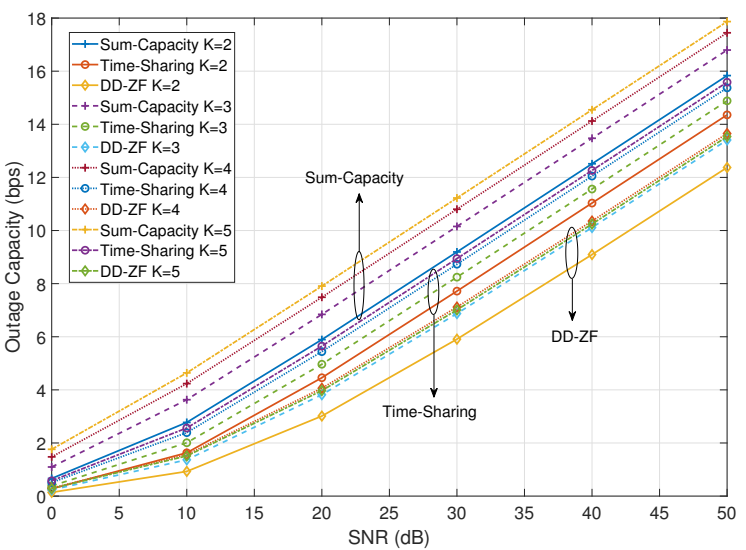

Fig. 14: Outage capacity of delay domain processing with perfect CSI.

The bit error rate (BER) performance of ZigZag algorithms is analyzed in Fig. 8 for both coded and uncoded cases with unit channel gains with perfect CSI and quadrature phase shift keying (QPSK) modulation. The channel coded results employ a rate $\frac{1}{2}$ low density parity check (LDPC) code of blocklength 4800. The results show that the double-zipper outperforms the original ZigZag in both uncoded and coded cases in a practical system. For instance, at a BER of $10^{-4}$, the double-zipper provides a performance gain of about $3 \mathrm{~dB}$ in both coded and uncoded cases over the original ZigZag. This can be due to the fact that in order to keep the complexity of the algorithm low, the noise correlation is not exploited when obtaining these results.

In order to have a more practical view, achievable rates of the time-sharing and the ZigZag schemes with finite alphabet signaling with quadrature amplitude modulation (QAM) are depicted in Fig. 11. As an example, for 16-QAM at an SNR of $40 \mathrm{~dB}$, at least $87 \%$ and $91 \%$ of the capacity of time-sharing can be achieved by the original and the double-zipper ZigZag algorithms, respectively. Clearly, the asymptotic performances of both ZigZag approaches are the same as the time-sharing scheme.

For the case of imperfect CSI, the outage and relative outage capacities are plotted versus SNR in Fig. 12 and Fig. 


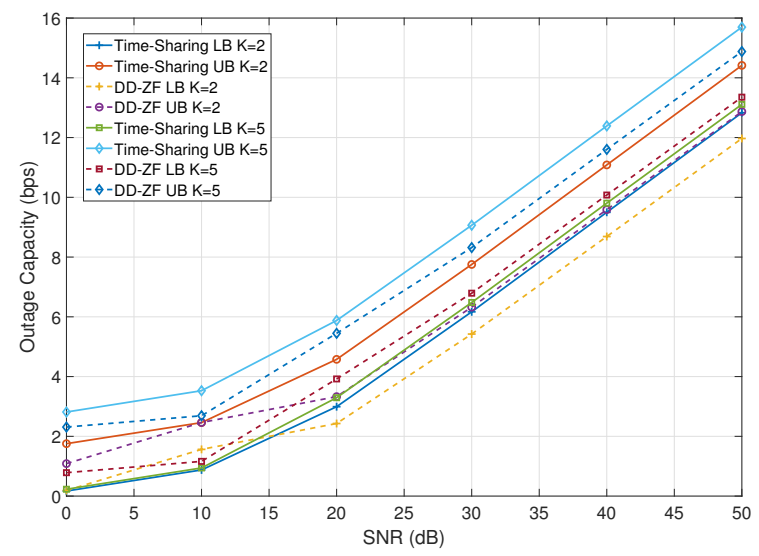

Fig. 15: Outage capacity of delay domain processing with imperfect CSI.

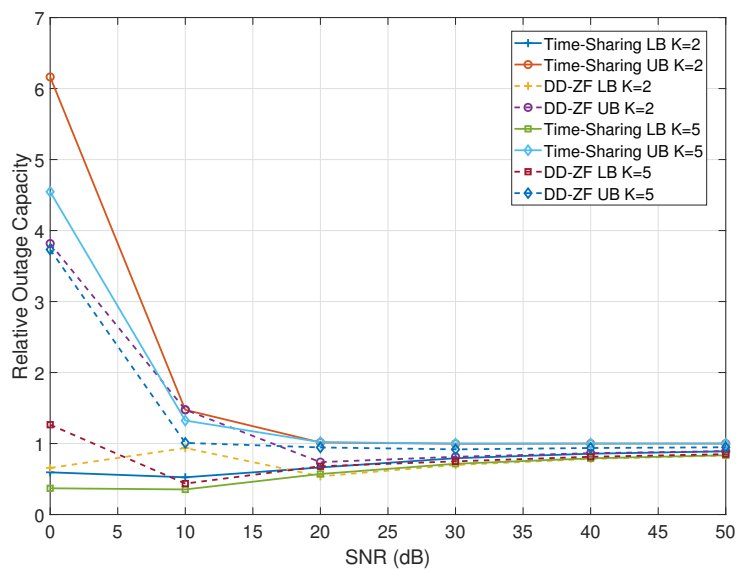

Fig. 16: Relative outage capacity of delay domain processing with imperfect CSI.

13 respectively. The relative capacities are normalized with respect to the time-sharing sum-rate capacity with perfect CSI. It can be seen that the capacity gaps of both ZigZag algorithms fade away in high SNRs, even with imperfect CSI.

Next we analyze the performance of the proposed delay domain approach. In Fig. 14, the outage capacity with perfect CSI is plotted versus the SNR. Interestingly, the performance of the DD-ZF does not improve that much as the number of users increases beyond two. This is due to the fact that additional time-intervals are required as the number of users increases. Also, the constant gap between the DD-ZF and the sum-capacity at high SNRs demonstrates the asymptotic optimality of this approach.

The outage and relative outage capacities for the case of imperfect CSI are plotted versus the SNR in Fig. 15 and Fig. 16 respectively. The relative capacities are normalized with respect to the time-sharing sum-rate capacity with perfect CSI. It can be seen that, similar to ZigZag, the capacity gaps fade away at high SNRs, even with imperfect CSI.

\section{CONCLUSIONS}

We present simple and scalable collision resolution algorithms based on the received signals in order to decouple the combined delayed packets of the users at the receiver in a multiple access setup. We first consider a two-user multiple access system with ZigZag decoupling at the receiver. We characterize the noise coefficient matrix and obtain performance bounds for both original and double-zipper ZigZag algorithms. Employing delay-domain processing, we then extend the results to arbitrary number of users by introducing a delaydomain zero forcing algorithm and its regularized variant. By deriving bounds on the achievable sum-rate of the proposed scheme for non-unit gain channels with imperfect CSI, we show that their performance approaches that of the optimal time-sharing scheme in the high SNR regime. These results are obtained without the need for any coordination, contrary to the time-sharing approach, and the performance is achieved by simple single user codes. Noting that there is no restriction on the codebooks, these algorithms can be employed in the setting of massive random access after combining them with suitable medium access protocols. We further note that we have made some idealistic assumptions such as perfect user activity estimation in our results. Analysis of the performance degradation due to more practical assumptions requires a deeper study, which we leave for future work.

\section{REFERENCES}

[1] M. Kazemi, T. M. Duman and M. Médard, "Double-zipper: multiple access with ZigZag decoding," 2020 IEEE Int. Conf. Commun. (ICC), Dublin, 2020, pp. 1-6.

[2] R. Ahlswede, "Multi-way communication channels," 2nd Int. Symp. Inf Theory (ISIT), Tsahkadsor, Armenia, USSR, 1971, pp. 23-52.

[3] H. Liao, "Multiple access channels," Ph.D. dissertation, Dept. Electr. Eng., Univ. Hawaii, Honolulu, 1972.

[4] T. M. Cover and J. A. Thomas. Elements of Information Theory. New York: Wiley, 1991.

[5] R. G. Gallager, "A perspective on multiaccess channels," IEEE Trans. Inf. Theory, vol. IT-31, pp. 124-142, Mar. 1985.

[6] R. Knopp, "Coding and multiple-access over fading channels," Ph.D. dissertation, EPFL, Lausanne, Switzerland, 1997.

[7] L. Liu, E. G. Larsson, W. Yu, P. Popovski, C. Stefanovic and E. de Carvalho, "Sparse signal processing for grant-free massive connectivity: A future paradigm for random access protocols in the internet of things," IEEE Signal Process. Mag., vol. 35, no. 5, pp. 88-99, Sept. 2018.

[8] A. Fengler, S. Haghighatshoar, P. Jung and G. Caire, "Non-Bayesian activity detection, large-scale fading coefficient estimation, and unsourced random access with a massive MIMO receiver," IEEE Trans. Inf. Theory, vol. 67, no. 5, pp. 2925-2951, May 2021.

[9] N. Abramson, "The ALOHA system-Another alternative for computer communications," in Proc. Fall 1970 AFIPS Comput. Conf., vol. 36, Houston, 1970, pp. 295-298.

[10] L. G. Roberts, "ALOHA packet system with and without slots and capture," ACM SIGCOMM Comput. Commun. Rev., vol. 5, no. 2, pp. $28-42,1975$.

[11] E. Paolini, G. Liva and M. Chiani, "Coded slotted ALOHA: A graphbased method for uncoordinated multiple access," IEEE Trans. Inf. Theory, vol. 61, no. 12, pp. 6815-6832, Dec. 2015.

[12] G. Choudhury and S. Rappaport, "Diversity ALOHA-A random access scheme for satellite communications," IEEE Trans. Commun., vol. 31, no. 3, pp. 450-457, Mar. 1983.

[13] E. Casini, R. De Gaudenzi, and O. del Rio Herrero, "Contention resolution diversity slotted ALOHA (CRDSA): An enhanced random access scheme for satellite access packet networks," IEEE Trans. Wireless Commun., vol. 6, no. 4, pp. 1408-1419, Apr. 2007.

[14] G. Liva, "Graph-based analysis and optimization of contention resolution diversity slotted ALOHA," IEEE Trans. Commun., vol. 59, no. 2, pp. 477-487, Feb. 2011.

[15] A. E. Mostafa, V. W. S. Wong, S. Liao, R. Schober, M. Ding and F. Wang, "Aggregate preamble sequence design for massive machine-type communications in 5G networks," in Proc. IEEE Global Commun. Conf. (GLOBECOM), Abu Dhabi, 2018, pp. 1-6. 
[16] Y. Polyanskiy, "A perspective on massive random-access," 2017 IEEE Int. Symp. Inf. Theory (ISIT), Aachen, 2017, pp. 2523-2527.

[17] O. Ordentlich and Y. Polyanskiy, "Low complexity schemes for the random access Gaussian channel," 2017 IEEE Int. Symp. Inf. Theory (ISIT), Aachen, 2017, pp. 2528-2532.

[18] A. Vem, K. R. Narayanan, J. Cheng and J. Chamberland, "A userindependent serial interference cancellation based coding scheme for the unsourced random access Gaussian channel," 2017 IEEE Inf. Theory Workshop (ITW), Kaohsiung, 2017, pp. 121-125.

[19] R. Calderbank and A. Thompson, "CHIRRUP: a practical algorithm for unsourced multiple access," arXiv:1811.00879v2, Dec. 2019.

[20] J. Hou, J. E. Smee, H. D. Pfister and S. Tomasin, "Implementing interference cancellation to increase the EV-DO Rev A reverse link capacity," IEEE Commun. Mag., vol. 44, no. 2, pp. 58-64, Feb. 2006.

[21] D. Truhachev and C. Schlegel, "Coupling data transmission for multipleaccess communications," IEEE Trans. Inf. Theory, vol. 65, no. 7, pp. 4550-4574, July 2019.

[22] S. Gollakota and D. Katabi, "ZigZag decoding: Combating hidden terminals in wireless networks," in Proc. ACM SIGCOMM, New York, 2008, pp. 159-170.

[23] J. Paek and M. J. Neely, "Mathematical analysis of throughput bounds in random access with ZigZag decoding," 2009 7th Int. Symp. Modeling and Optimization in Mobile, Ad Hoc, and Wirel. Netw. (WiOpt), Seoul, 2009, pp. 1-7.

[24] A. ParandehGheibi, J. K. Sundararajan and M. Médard, "Collision helps - Algebraic collision recovery for wireless erasure networks," 2010 Third IEEE Int. Workshop Wirel. Netw. Coding (WiNC), Boston, 2010, pp. 1-6.

[25] A. ParandehGheibi, J. K. Sundararajan and M. Médard, "Acknowledgement design for collision-recovery-enabled wireless erasure networks," 2010 48th Annu. Allerton Conf. Commun., Control, and Comput. (Allerton), Allerton, 2010, pp. 435-442.

[26] A. S. Tehrani, A. G. Dimakis and M. J. Neely, "SigSag: Iterative detection through soft message-passing," IEEE J. Sel. Topics Signal Process., vol. 5, no. 8, pp. 1512-1523, Dec. 2011.

[27] C. W. Sung and X. Gong, "A ZigZag-decodable code with the MDS property for distributed storage systems," 2013 IEEE Int. Symp. Inf. Theory (ISIT), Istanbul, 2013, pp. 341-345.

[28] T. Nozaki, "Fountain codes based on ZigZag decodable coding," 2014 Int. Symp. Inf. Theory and its Appl. (ISITA), Melbourne, 2014, pp. 274278.

[29] S. Ogata and K. Ishibashi, "Application of ZigZag decoding in frameless ALOHA," IEEE Access, vol. 7, pp. 39528-39538, 2019.

[30] M. Oinaga, S. Ogata and K. Ishibashi, "Design of Coded ALOHA With ZigZag Decoder," IEEE Access, vol. 7, pp. 168527-168535, 2019.

[31] L. Bai, R. Han, J. Liu, J. Choi and W. Zhang, "Multiple delay estimation for collision resolution in non-orthogonal random access," IEEE Trans. Veh. Technol., vol. 69, no. 1, pp. 497-508, Jan. 2020.

[32] A. S. Avestimehr, S. N. Diggavi and D. N. C. Tse, "Wireless network information flow: A deterministic approach," IEEE Trans. Inf. Theory, vol. 57, no. 4, pp. 1872-1905, Apr. 2011.

[33] P. Aquilina and T. Ratnarajah, "Performance analysis of IA techniques in the MIMO IBC with imperfect CSI," IEEE Trans. Commun., vol. 63 , no. 4, pp. 1259-1270, Apr. 2015.

[34] T. Yoo and A. Goldsmith, "Capacity and power allocation for fading MIMO channels with channel estimation error," IEEE Trans. Inf. Theory, vol. 52, no. 5, pp. 2203-2214, May 2006.

[35] M. Médard, "The effect upon channel capacity in wireless communications of perfect and imperfect knowledge of the channel," IEEE Trans. Inf. Theory, vol. 46, no. 3, pp. 933-946, May 2000.

[36] A. El Gamal and Y. H. Kim, Network Information Theory. Cambridge: Cambridge University Press, 2011.

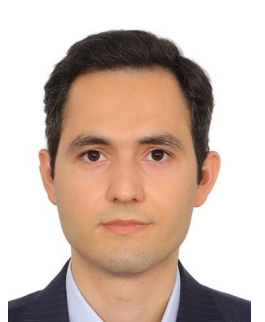

Mohammad Kazemi (S'14-M'18) received the B.S. and M.S. degrees from the K.N. Toosi University of Technology (Tehran, Iran) in 2007 and 2010, respectively, and the Ph.D. degree from Amirkabir University of Technology (Tehran, Iran) in 2017, all in electrical engineering. He was a visiting student with Bilkent University, Turkey, in 2015. He was a researcher in MMWCL at Amirkabir University of Technology from 2017 to 2019 . He is now a postdoctoral fellow in the EE Department at Bilkent University. His research interests are in wireless communications and signal processing, with particular focus on massive MIMO and massive random access systems. He is currently the Editorial Assistant to the Editor-in-Chief of IEEE Transactions on Communications.

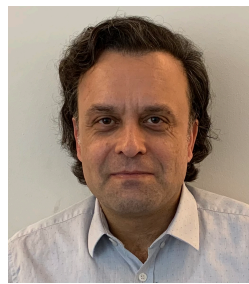

Tolga M. Duman (S'95-M'98-SM'03-F'11) is a Professor of Electrical and Electronics Engineering Department at Bilkent University, in Turkey. He received the B.S. degree from Bilkent University, Ankara, Turkey, in 1993, and the M.S. and Ph.D. degrees from Northeastern University, Boston, MA, USA, in 1995 and 1998, respectively, all in electrical engineering. Prior to joining Bilkent University in September 2012, he was a Professor with the School of ECEE at Arizona State University. His current research interests are in systems, with particular focus on communication and signal processing, including wireless and mobile communications, coding/modulation, coding for wireless communications, and machine learning. Dr. Duman is a recipient of the National Science Foundation CAREER Award and the IEEE Third Millennium Medal, and he is currently the Editor-in-Chief of IEEE Transactions on Communications.

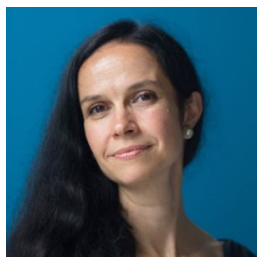

Muriel Médard (Fellow, IEEE) is the Cecil $\mathrm{H}$. Green Professor with the Electrical Engineering and Computer Science (EECS) Department, MIT and leads the Network Coding and Reliable Communications Group, Research Laboratory for Electronics, MIT. She is a member of the American Academy of Arts and Sciences, the US National Academy of Engineering and a Fellow of the US National Academy of Inventors. She received the 2016 IEEE Vehicular Technology James Evans Avant Garde Award, the 2017 Aaron Wyner Distinguished Service Award from the IEEE Information Theory Society, and the 2017 IEEE Communications Society Edwin Howard Armstrong Achievement Award. She received the 2019 Best Paper Award for IEEE Transactions on Network Science and Engineering, the 2009 IEEE Communication Society and Information Theory Society Joint Paper Award, the 2009 William R. Bennett Prize in the Field of Communications Networking, the 2002 IEEE Leon K. Kirchmayer Prize Paper Award, the 2018 ACM SIGCOMM Test of Time Paper Award, and several conference paper awards. She was Co-Winner of the MIT 2004 Harold E. Egerton Faculty Achievement Award, and received the 2013 EECS Graduate Student Association Mentor Award. She served as undergraduate Faculty in Residence for seven years. For technology transfer, she has co-founded two companies, CodeOn, for which she consults, and Steinwurf, for which she is a Chief Scientist. She was a President of the IEEE Information Theory Society in 2012, and served on its board of governors for 12 years. She has served as a technical program committee co-chair of many of the major conferences in information theory, communications, and networking. She has served as associate editor and guest editor for many IEEE publications, as well as the Editor-in-Chief of the IEEE Journal on Selected Areas in Communications and currently serves as Editor-in-Chief of the IEEE Transactions on Information Theory. 